\title{
onewaytests: An R Package for One-Way Tests in Independent Groups Designs
}

\author{
by Osman Dag, Anil Dolgun and Naime Meric Konar
}

\begin{abstract}
One-way tests in independent groups designs are the most commonly utilized statistical methods with applications on the experiments in medical sciences, pharmaceutical research, agriculture, biology, engineering, social sciences and so on. In this paper, we present the onewaytests package to investigate treatment effects on the dependent variable. The package offers the one-way tests in independent groups designs, which include ANOVA, Welch's heteroscedastic F test, Welch's heteroscedastic $F$ test with trimmed means and Winsorized variances, Brown-Forsythe test, AlexanderGovern test, James second order test and Kruskal-Wallis test. The package also provides pairwise comparisons, graphical approaches, and assesses variance homogeneity and normality of data in each group via tests and plots. A simulation study is also conducted to give recommendations for applied researchers on the selection of appropriate one-way tests under assumption violations. Furthermore, especially for non-R users, a user-friendly web application of the package is provided. This application is available at http://www. softmed. hacettepe. edu. tr/onewaytests.
\end{abstract}

\section{Introduction}

There are many statistical procedures for one-way independent groups designs. The one-way fixed effects analysis of variance (ANOVA) is the most common one being used for comparing $k$ independent group means. Independence of the observations in each group and between groups, normality (i.e., $k$ samples come at random from normal distribution) and variance homogeneity (i.e., $k$ populations have equal variances) are three basic assumptions of ANOVA. ANOVA is a valid and powerful test for identifying group differences provided that these assumptions are held. However, most of the data sets in practice are not normally distributed and group variances are heterogeneous, and it is difficult to find a data set that satisfies both assumptions. When the assumptions underlying ANOVA are violated, the drawn inferences are also invalid. There have been remarkable efforts to find an appropriate and robust test statistic under non-normality and variance heterogeneity. Several procedures alternative to ANOVA were proposed, including Welch's heteroscedastic $F$ test (Welch, 1951), Welch's heteroscedastic $F$ test with trimmed means and Winsorized variances (Welch, 1951), Alexander-Govern test (Alexander and Govern, 1994), James second order test (James, 1951, 1954), Brown-Forsythe test (Brown and Forsythe, 1974a,b) and Kruskal-Wallis test (Kruskal and Wallis, 1952).

In the literature, there are many studies in which the comparison of ANOVA and its alternative tests was made with respect to type I error rate and power when the assumptions of ANOVA are not satisfied. The effects of sample size (balanced/unbalanced case), non-normality, unequal variances, and combined effects of non-normality and unequal variances were investigated in details (Wilcox, 1988; Cribbie et al., 2012; Lantz, 2013; Gamage and Weerahandi, 1998; Parra-Frutos, 2013; Cribbie et al., 2007). In the light of these studies, we conduct a Monte Carlo simulation study in an attempt to give recommendations for applied researchers on the selection of appropriate one-way tests.

ANOVA, Welch's heteroscedastic $F$ test, Welch's heteroscedastic $F$ test with trimmed means and Winsorized variances, Kruskal-Wallis test, and Brown-Forsythe test are available under some packages (given in Table 1) on the Comprehensive R Archive Network (CRAN). Alexander-Govern test and James second order test are also found to be robust to assumption violations, but have been overlooked as their calculation and implementation were not easily available elsewhere.

In this paper, we introduce an R package, onewaytests (Dag et al., 2017) which implements Alexander-Govern test and James second order test, in addition to the ANOVA, Welch's heteroscedastic $F$ test, Welch's heteroscedastic $F$ test with trimmed means and Winsorized variances, Kruskal-Wallis test, and Brown-Forsythe test. Besides the omnibus tests, the package provides pairwise comparisons to investigate which groups create the difference providing that a statistically significant difference is obtained by the omnibus test. Moreover, the package offers several graphic types such as grouped box-and-whisker plot and error bar graph. Also, it provides statistical tests and plots (i.e. Q-Q plot and histogram) to assess variance homogeneity and normality, in the package version 1.5 and later. The onewaytests package is publicly available on the CRAN.

The organization of this paper is presented as follows. First, we give the theoretical background on the one-way tests in independent groups designs. Second, we introduce the onewaytests package and demonstrate the applicability of the package using two real-life datasets. Third, the web interface of the onewaytests package is introduced. A Monte Carlo simulation study is also conducted to give recommendations for applied researchers on the selection of appropriate tests included in the package. 
Results of this simulation study and the general conclusions on the effects of assumption violations are mentioned.

\section{One-way tests in independent groups designs}

In this section, the statistical tests that are used to test the equality of several populations in the oneway independent groups designs are explained. Among these tests, ANOVA, Welch's heteroscedastic $F$ test (Welch, 1951), Alexander-Govern test (Alexander and Govern, 1994), James second order test (James, 1951, 1954) and Brown-Forsythe test (Brown and Forsythe, 1974a,b) test the null hypothesis $H_{0}: \mu_{1}=\mu_{2}=\ldots=\mu_{k}$ versus alternative $H_{1}:$ at least one $\mu_{j}(j=1,2, \ldots, k)$ is different. Welch's heteroscedastic $F$ test with trimmed means and Winsorized variances (Welch, 1951) tests the equality of population trimmed means, $H_{0}: \mu_{t 1}=\mu_{t 2}=\ldots=\mu_{t k}$ versus alternative $H_{1}$ : at least one $\mu_{t j}$ is different, where $\mu_{t j}$ represents the trimmed mean of the $j$ th population $(j=1,2, \ldots, k)$. Kruskal-Wallis test (Kruskal and Wallis, 1952) tests the null hypothesis $H_{0}: \theta_{1}=\theta_{2}=\ldots=\theta_{k}$ versus alternative $H_{1}$ : at least one $\theta_{j}(j=1,2, \ldots, k)$ is different. For the Kruskal-Wallis test, $\theta_{j}$ represents sum of the ranks of the $j$ th population.

\section{ANOVA}

The one-way fixed effects analysis of variance (ANOVA) is the most common statistical procedure to test the equality of $k$ independent population means. Underlying assumptions associated with ANOVA include homogeneity of group variances, normality of distributions and statistical independence of errors. Under these conditions, the ANOVA test statistic,

$$
F=\frac{\sum_{j} n_{j}\left(\bar{X}_{. j}-\bar{X}_{. . .}\right)^{2} /(k-1)}{\sum_{i} \sum_{j}\left(X_{i j}-\bar{X}_{. j}\right)^{2} /(N-k)}
$$

follows an $F$ distribution with $k-1$ degrees of freedom for the numerator and $N-k$ degrees of freedom for the denominator. In Equation (1), $k$ is the number of groups, $N$ is the total number of observations, and $n_{j}$ is the number of observations in the jth group. $X_{i j}$ is the $i$ th observation $\left(i=1,2, \ldots, n_{j}\right)$ in the $j$ th group $(j=1,2, \ldots, k), \sum_{j} n_{j}=N, \bar{X}_{\text {.. }}$ is the overall mean, where $\bar{X}_{. .}=\sum_{j} n_{j}\left(\bar{X}_{. j}\right) / N$ and $\bar{X}_{. j}$ is sample mean for the $j$ th group, where $\bar{X}_{. j}=\sum_{i} X_{i j} / n_{j}$. ANOVA is more powerful if the assumptions of normality and variance homogeneity hold true. Non-normality has minimal effect on the type I error when the variances are equal (Lantz, 2013) but when the variances are not equal, ANOVA provides poor control over the type I and type II error rates (Bishop, 1976).

\section{Welch's heteroscedastic $F$ test}

Welch (1951) proposed a heteroscedastic alternative to ANOVA that is robust to the violation of variance homogeneity assumption. Under the null hypothesis $H_{0}: \mu_{1}=\mu_{2}=\ldots=\mu_{k}$, the test statistic $F_{w}$,

$$
F_{w}=\frac{\sum_{j} w_{j}\left(\bar{X}_{. j}-X_{. .}^{\prime}\right)^{2} /(k-1)}{\left[1+\frac{2}{3}((k-2) v)\right]},
$$

follows an $F$ distribution with degrees of freedom $k-1$ for the numerator and $1 / v$ degrees of freedom for the denominator. In Equation (2), $w_{j}=n_{j} / S_{j}^{2}, S_{j}^{2}=\sum_{i}\left(X_{i j}-\bar{X}_{. j}\right)^{2} /\left(n_{j}-1\right)$,

$$
X_{. .}^{\prime}=\frac{\sum_{j} w_{j} \bar{X}_{. j}}{\sum_{j} w_{j}}
$$

and

$$
v=\frac{3 \sum_{j}\left[\left(1-\frac{w_{j}}{\sum_{j} w_{j}}\right)^{2} /\left(n_{j}-1\right)\right]}{k^{2}-1} .
$$

Welch's heteroscedastic $F$ test is robust and less sensitive to heteroscedasticity when compared to the ANOVA as within groups variance is based on the relationship between the different sample sizes in the different groups instead of a simple pooled variance estimate (Lantz, 2013). 


\section{Welch's heteroscedastic $F$ test with trimmed means and Winsorized variances}

Welch's heteroscedastic $F$ test with trimmed means and Winsorized variances (Welch, 1951) is a robust procedure that tests the equality of means by substituting trimmed means and Winsorized variances for the usual means and variances. Therefore, this test statistic is relatively insensitive to the combined effects of non-normality and variance heterogeneity (Keselman et al., 2008). Let $X_{(1) j} \leq X_{(2) j} \leq \ldots \leq X_{\left(n_{j}\right) j}$ be the ordered observations in the $j$ th group and $g_{j}=\left\|\epsilon n_{j}\right\|, \epsilon$ is the proportion to be trimmed in each tail of the distribution. After trimming, the effective sample size for the $j$ th group becomes $h_{j}=n_{j}-2 g_{j}$. Then $j$ th sample trimmed mean is

$$
\bar{X}_{t j}=\frac{1}{h_{j}} \sum_{i=g_{j}+1}^{n_{j}-g_{j}} X_{(i) j},
$$

and $j$ th sample Winsorized mean is

$$
\bar{X}_{w j}=\frac{1}{n_{j}} \sum_{i=1}^{n_{j}} Y_{i j}
$$

where

$$
Y_{i j}= \begin{cases}X_{\left(g_{j}+1\right) j} & \text { if } X_{i j} \leq X_{\left(g_{j}+1\right) j}, \\ X_{i j} & \text { if } X_{\left(g_{j}+1\right) j}<X_{i j}<X_{\left(n_{j}-g_{j}\right) j}, \\ X_{\left(n_{j}-g_{j}\right) j} & \text { if } X_{i j} \geq X_{\left(n_{j}-g_{j}\right) j} .\end{cases}
$$

The sample Winsorized variance is

$$
s_{w j}^{2}=\frac{1}{\left(n_{j}-1\right)} \sum_{i=1}^{n_{j}}\left(Y_{i j}-\bar{X}_{w j}\right)^{2} .
$$

Let

$$
\begin{gathered}
q_{j}=\frac{\left(n_{j}-1\right) s_{w j}^{2}}{h_{j}\left(h_{j}-1\right)}, \\
w_{j}=\frac{1}{q_{j}}, \\
U=\sum_{j} w_{j}, \\
\tilde{X}=\frac{1}{U} \sum_{j} w_{j} \bar{X}_{t j}, \\
A=\frac{1}{k-1} \sum_{j} w_{j}\left(\bar{X}_{t j}-\tilde{X}\right)^{2}, \\
B=\frac{2(k-2)}{k^{2}-1} \sum_{j} \frac{\left(1-w_{j} / U\right)^{2}}{h_{j}-1} .
\end{gathered}
$$

Under $H_{0}: \mu_{t 1}=\mu_{t 2}=\ldots=\mu_{t k}$, Welch's heteroscedastic $F$ test with trimmed means and Winsorized variances $F_{w t}$,

$$
F_{w t}=\frac{A}{B+1},
$$

follows an approximately $F$ distribution with $k-1$ and $v^{\prime}$ degrees of freedom, where $v^{\prime}$ is

$$
v^{\prime}=\left(\frac{3}{k^{2}-1} \sum_{j} \frac{\left(1-w_{j} / U\right)^{2}}{h_{j}-1}\right)^{-1} .
$$

$F_{w t}$ is not only less sensitive to heteroscedasticity and non-normality but also robust to the negative effects of outliers as it utilizes the trimmed means and Winsorised variances (Keselman et al., 2008). 


\section{Brown-Forsythe test}

Brown and Forsythe $(1974 a, b)$ proposed the following test statistic:

$$
F_{B F}=\frac{\sum_{j} n_{. j}\left(\bar{X}_{. j}-\bar{X}_{. .}\right)^{2}}{\sum_{j}\left(1-n_{j} / N\right) S_{j}^{2}} .
$$

Under the null hypothesis, $F_{B F}$ statistic has an approximately $F$ distribution with $k-1$ and $f$ degrees of freedom, where $f$ is obtained with

$$
f=\left(\sum_{j} c_{j}^{2} /\left(n_{j}-1\right)\right)^{-1},
$$

and

$$
c_{j}=\frac{\left(1-n_{j} / N\right) S_{j}^{2}}{\left[\sum_{j}\left(1-n_{j} / N\right) S_{j}^{2}\right]} .
$$

$F_{B F}$ is a modification of ANOVA which has the same numerators as the ANOVA but an altered denominator. This test is more powerful when some variances appear unusually low (Brown and Forsythe, 1974a).

\section{Alexander-Govern test}

Alexander and Govern (1994) presented another robust test that is alternative to ANOVA, and it is used when group variances are not homogeneous. The test statistic for Alexander-Govern test is

$$
\chi_{A G}^{2}=\sum_{j=1}^{k} z_{j}^{2} .
$$

Under the null hypothesis, $\chi_{A G}^{2}$ is distributed as $\chi^{2}$ distribution with $k-1$ degrees of freedom. In Equation (5),

$$
z_{j}=c+\frac{\left(c^{3}+3 c\right)}{b}-\frac{\left(4 c^{7}+33 c^{5}+240 c^{3}+855 c\right)}{\left(10 b^{2}+8 b c^{4}+1000 b\right)},
$$

where $c=\left[\alpha \times \ln \left(1+t_{j}^{2} / v_{j}\right)\right]^{1 / 2}, b=48 \alpha^{2}, \alpha=v_{j}-0.5$ and $v_{j}=n_{j}-1$. The $t$ statistic for each group is

$$
t_{j}=\frac{\bar{X}_{. j}-X^{+}}{S_{j}^{\prime}} .
$$

The variance-weighted mean is $X^{+}=\sum_{j=1}^{k} w_{j} \bar{X}_{. j}$ and the standard error for the $j$ th group is

$$
S_{j}^{\prime}=\left[\frac{\sum_{i=1}^{n_{j}}\left(X_{i j}-\bar{X}_{. j}\right)^{2}}{n_{j}\left(n_{j}-1\right)}\right]^{1 / 2} .
$$

Also, weights for each group are defined as

$$
w_{j}=\frac{1 / S_{j}^{\prime 2}}{\sum_{j=1}^{k} 1 / S_{j}^{\prime 2}} .
$$

$\chi_{A G}^{2}$ is another modification of ANOVA under heterogeneity of variance using the normalizing transformation of the one-sample $t$ statistic. This test provides a good control of type I and II error rates for normally distributed data, but it is not robust to non-normality (Myers, 1998).

\section{James second order test}

An alternative test to ANOVA was proposed by James (1951). This test statistic $(J)$ is

$$
J=\sum_{j} t_{j}^{2}
$$


where $t_{j}$ is given in Equation (6). The test statistic, $J$, is compared to a critical value, $h(\alpha)$, where

$$
\begin{aligned}
h(\alpha) & =r+\frac{1}{2}\left(3 \chi_{4}+\chi_{2}\right) T+\frac{1}{16}\left(3 \chi_{4}+\chi_{2}\right)^{2}\left(1-\frac{k-3}{r}\right) T^{2} \\
& +\frac{1}{2}\left(3 \chi_{4}+\chi_{2}\right)\left(8 R_{23}-10 R_{22}+4 R_{21}-6 R_{12}^{2}+8 R_{12} R_{11}-4 R_{11}^{2}\right) \\
& +\left(2 R_{23}-4 R_{22}+2 R_{21}-2 R_{12}^{2}+4 R_{12} R_{11}-2 R_{11}^{2}\right)\left(\chi_{2}-1\right) \\
& +\frac{1}{4}\left(-R_{12}^{2}+4 R_{12} R_{11}-2 R_{12} R_{10}-4 R_{11}^{2}+4 R_{11} R_{10}-R_{10}^{2}\right)\left(3 \chi_{4}-2 \chi_{2}-1\right) \\
& +\left(R_{23}-3 R_{22}+3 R_{21}-R_{20}\right)\left(5 \chi_{6}+2 \chi_{4}+\chi_{2}\right) \\
& +\frac{3}{16}\left(R_{12}^{2}-4 R_{23}+6 R_{22}-4 R_{21}+R_{20}\right)\left(35 \chi_{8}+15 \chi_{6}+9 \chi_{4}+5 \chi_{2}\right) \\
& +\frac{1}{16}\left(-2 R_{22}+4 R_{21}-R_{20}+2 R_{12} R_{10}-4 R_{11} R_{10}+R_{10}^{2}\right)\left(9 \chi_{8}-3 \chi_{6}-5 \chi_{4}-\chi_{2}\right) \\
& +\frac{1}{4}\left(-R_{22}+R_{11}^{2}\right)\left(27 \chi_{8}+3 \chi_{6}+\chi_{4}+\chi_{2}\right)+\frac{1}{4}\left(R_{23}-R_{12} R_{11}\right)\left(45 \chi_{8}+9 \chi_{6}+7 \chi_{4}+3 \chi_{2}\right) .
\end{aligned}
$$

For any integers $s$ and $t, R_{s t}=\sum_{j}\left(n_{j}-1\right)^{-s} w_{j}^{t}, \chi_{2 s}=r^{s} /[(k-1)(k+1) \ldots(k+2 s-3)]$ where $r$ is the $(1-\alpha)$ centile of a $\chi^{2}$ distribution with $k-1$ degrees of freedom, $T=\sum_{j}\left(1-w_{j}\right)^{2} /\left(n_{j}-1\right)$ and $w_{j}$ is defined in Equation (7). If the test statistic, J, exceeds $h(\alpha)$, then the null hypothesis is rejected.

The James second order test has been acknowledged as the best option for both normal heteroscedastic data (Alexander and Govern, 1994) and non-normal symmetric heteroscedastic data (Oshima and Algina, 1992). The disadvantage of this method is the complexity of the computation of critical values.

\section{Kruskal-Wallis test}

Kruskal and Wallis (1952) proposed the nonparametric alternative to ANOVA. Let $r_{i j}$ denote the rank of $X_{i j}$ when $N=n_{1}+\ldots+n_{k}$ observations are ranked from smallest to largest. $R_{j}=\sum_{i=1}^{n_{j}} r_{i j}$ is the sum of ranks assigned to the observations in the $j$ th group and $\bar{R}_{j}=R_{j} / n_{j}$ is the average rank for these observations. Under these definitions, the Kruskal-Wallis test statistic is given by

$$
\chi_{K W}^{2}=\frac{1}{S^{2}}\left(\sum_{j=1}^{k} \frac{R_{j}^{2}}{n_{j}}-\frac{N(N+1)^{2}}{4}\right),
$$

where

$$
S^{2}=\frac{1}{N-1}\left(\sum_{j=1}^{k} \sum_{i=1}^{n_{j}} r_{i j}^{2}-\frac{N(N+1)^{2}}{4}\right) .
$$

When $n_{j} \rightarrow \infty, \chi_{K W}^{2}$ has an asymptotic $\chi^{2}$ distribution under the null hypothesis $H_{0}: \theta_{1}=\theta_{2}=$ $\ldots=\theta_{k}$. We reject $H_{0}$ if $\chi_{K W}^{2} \geq \chi_{k-1, \alpha}^{2}$ where $\chi_{k-1, \alpha}^{2}$ is the upper $\alpha$ percentile for the $\chi^{2}$ distribution with $k-1$ degrees of freedom. Note that, when there are no ties, $S^{2}$ simplifies to $N(N+1) / 12$.

The Kruskal-Wallis test is robust to non-normality as it utilizes ranks instead of actual values. However, it assumes that the observations in each group come from populations with the same shape of distribution. Therefore, if different groups have different shapes (e.g., some are skewed to the right and another is skewed to the left), the Kruskal-Wallis test may give inaccurate results (Fagerland and Sandvik, 2009).

There are other R packages including one-way tests in independent groups designs; namely, stats (R Core Team, 2017), lawstat (Gastwirth et al., 2017), coin (Hothorn et al., 2006), car (Fox and Weisberg, 2011), WRS2 (Mair et al., 2017), welchADF (Villacorta, 2017). The aov . test (onewaytests) can be seen as a simplified version of anova (stats) and Anova (car), but the latter two are more flexible and comprehensive in a way that they can handle two or more variables (e.g., two-way ANOVA, multivariate ANOVA, repeated measures ANOVA, etc.). Amongst the alternatives, the onewaytests is the first package including seven different one-way independent design tests that are covered in one package. A brief comparison between these packages and onewaytests is given in Table 1 . In Table 1, the numbers in parentheses indicate the rankings of the functions in terms of computational speed and the checks indicate the availability of the specific one-way design test in the packages. The 
onewaytests package has the fastest running times except for Welch's heteroscedastic $F$ test, where the stats's function elapse time is 0.001 seconds faster. Note that the computational time is system-specific and may vary according to the number of iterations and the inclusion of additional arguments in the functions.

Table 1: Comparison of $\mathrm{R}$ packages including one-way independent design tests

\begin{tabular}{lccccccc}
\hline Test & stats & car & lawstat & welchADF & WRS2 & coin & onewaytests \\
\hline$F$ & $(2) \checkmark$ & $(3) \checkmark$ & & & & & $(1) \checkmark$ \\
$F_{w}$ & $(1) \checkmark$ & & & $(4) \checkmark$ & $(3) \checkmark$ & & $(2) \checkmark$ \\
$F_{w t}$ & & & $(2) \checkmark$ & $(3) \checkmark$ & $(2) \checkmark$ & $(1) \checkmark$ \\
$F_{B F}$ & & & & & & & $(1) \checkmark$ \\
$\chi_{A G}^{2}$ & & & & & & & $\checkmark$ \\
$J$ & & & & $(3) \checkmark$ & $(1) \checkmark$ \\
$\chi_{K W}^{2}$ & $(2) \checkmark$ & & & & & & \\
\hline
\end{tabular}

\section{Demonstration of the onewaytests package}

The onewaytests package includes several functions specially designed for one-way independent groups designs along with functions for assessing fundamental assumptions via relevant tests and plots. In this section, we demonstrate the usage of onewaytests package by using two different data sets from different fields.

\section{Iris data}

In this part, we work with iris data set, collected by Anderson (1935), available in R. Fisher (1936) introduced this data set as an application of the linear discriminant analysis. At present, the popularity of the data set still continues within a variety of studies; examples include the studies related to data mining (Hu, 2005), multivariate normality assessment (Korkmaz et al., 2014), and so on.

This data set includes iris flowers' length and width measurements of sepal and petal characteristics in centimeters along with a grouping variable indicating the type of iris flowers (setosa, virginica and versicolor). For illustrating the implementation of our package, we use sepal length measurements as a response variable and iris types as a grouping variable. This data set has a total of 150 observations (50 observations in each type of iris flowers).

After installing and loading onewaytests package, the functions designed for one-way independent groups designs are available to be used. One-way tests in this package generally give test statistics and p-values to decide on the hypothesis of the statistical process, except for James second order test. In this test, test statistic and critical value are given as an output since the asymptotic distribution of the test statistic is not available.

The onewaytests package is also able to give some basic descriptive statistics of the given groups.

\# obtain some basic descriptive statistics

$\mathrm{R}>$ describe (Sepal.Length $\sim$ Species, data = iris)

\begin{tabular}{|c|c|c|c|c|c|c|c|c|c|c|c|}
\hline & $\mathrm{n}$ & Mean & Std.Dev & Median & Min & $\operatorname{Max}$ & 25 th & 75 th & Skewness & Kurtosis & NA \\
\hline setosa & 50 & 5.006 & 0.3524897 & 5.0 & 4.3 & 5.8 & 4.800 & 5.2 & 0.1164539 & 2.654235 & \\
\hline versicolor & 50 & 5.936 & 0.5161711 & 5.9 & 4.9 & 7.0 & 5.600 & 6.3 & 0.1021896 & 2.401173 & \\
\hline virginica & 50 & 6.588 & 0.6358796 & 6.5 & 4.9 & 7.9 & 6.225 & 6.9 & 0.1144447 & 2.912058 & \\
\hline
\end{tabular}

For all functions in the onewaytests package, the argument must be specified as a formula in which left and right-hand sides of the formula give sample values and its corresponding groups, respectively. The left and right-hand sides of the formula must have one variable. The variable on the left must be a numeric while the variable on the right must be a factor. Otherwise, each function returns an error message.

The functions for one-way design tests are coded from scratch. The function for pairwise comparison is coded for pairwise comparison of one-way independent groups designs by using the p-value adjustment methods available in the $\mathrm{p}$. adjust function under stats package. For checking assumptions, the tests are used from different packages (stats, car and nortest (Gross and Ligges, 2015)) and adapted for one-way independent groups designs. The function for the graphics is coded from scratch using the ggplot2 package (Wickham, 2009). 


\section{One-way tests in independent groups designs}

The onewaytests package includes seven one-way tests in independent groups designs. In this part, the implementations of these tests are presented.

\section{ANOVA: aov.test(...)}

The aov. test is utilized to test the equality of $k$ independent population means.

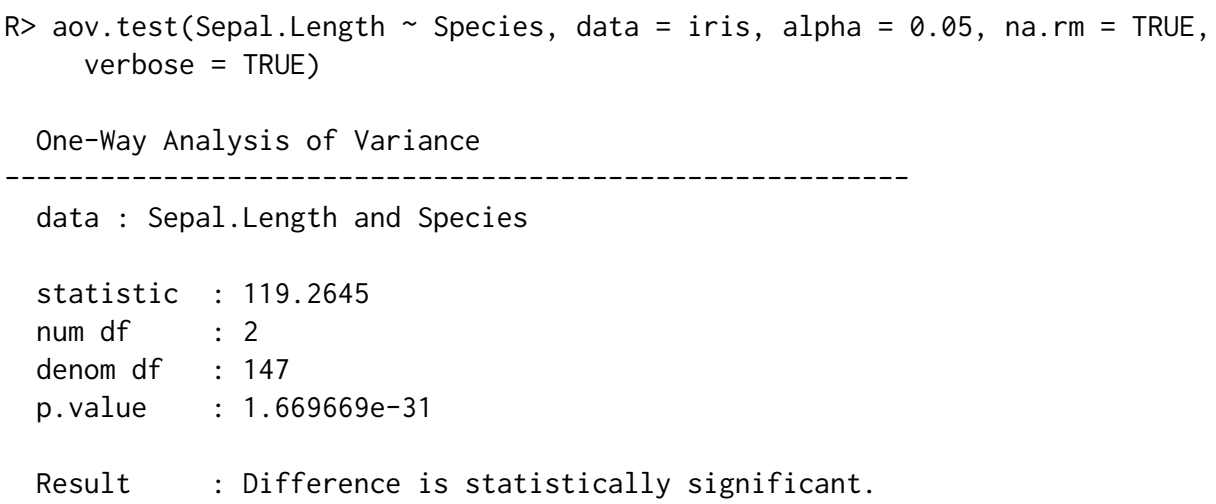

Here, the statistic is the ANOVA test statistic distributed as F with the degrees of freedom for the numerator (num $d f$ ) and denominator (denom $d f$ ). Also, $p$. value is the significance value of the test statistic. Since the p-value, derived from aov. test, is lower than 0.05 , it can be concluded that there is statistically significant difference between the iris species $\left(\mathrm{F}=119.2645\right.$, $\mathrm{p}$-value $\left.=1.669669 \times 10^{-31}\right)$.

alpha is the level of significance to assess the statistical difference. Default is set to alpha $=0.05$. na. $r$ is a logical value indicating whether NA values should be stripped before the computation proceeds. Default is na. $r m=$ TRUE. verbose is a logical for printing output to R console. Default is set to verbose $=$ TRUE. These arguments are available in the functions for one-way tests and checking assumptions. The users who would like to use the statistics in the output in their programs can use the following codes.

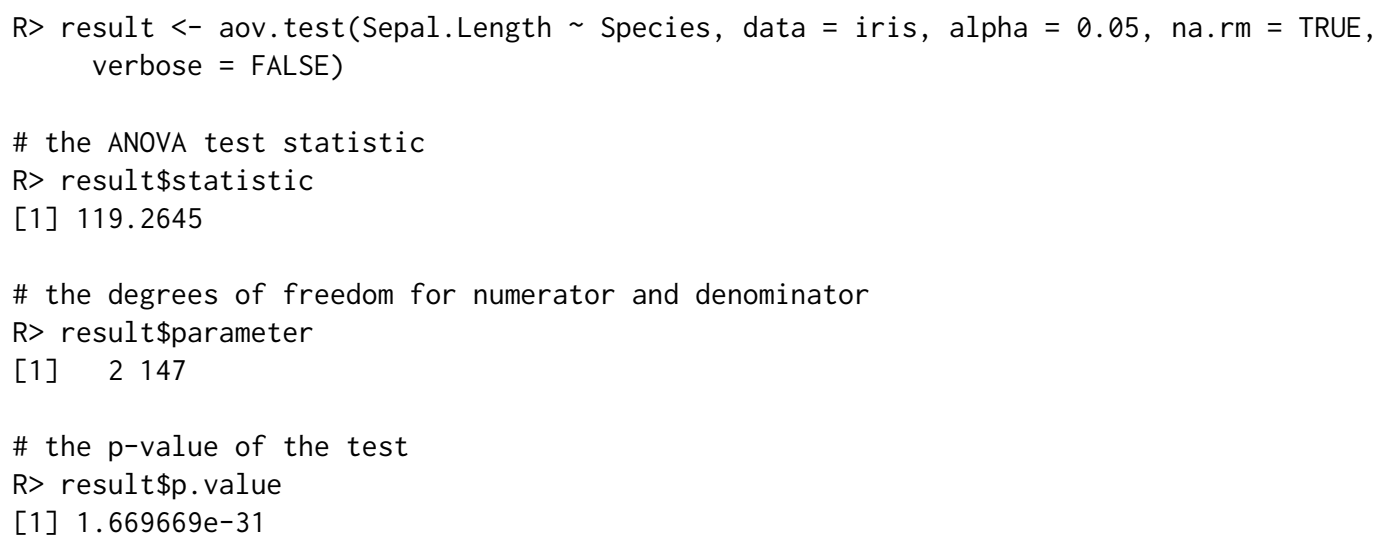

Here, the codes for how to obtain the statistics from the ANOVA output are given. Since all one-way design tests return similar outputs, similar codes are not repeated in the other tests. For all tests, the level of significance is taken as 0.05 .

\section{Welch's heteroscedastic $F$ test: welch.test(...)}

One may use the welch. test function in the onewaytests package to perform Welch's heteroscedastic $F$ test.

R> welch.test (Sepal.Length $\sim$ Species, data $=$ iris)

Welch's Heteroscedastic F Test

data : Sepal.Length and Species 


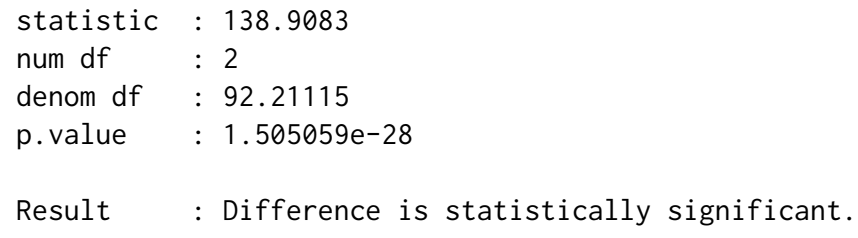

In the output, similar to aov. test, the statistic is the Welch's test statistic distributed as F with the degrees of freedom for the numerator (num $d f$ ) and denominator (denom $d f$ ). One can conclude that the difference between the species is statistically significant $\left(\mathrm{F}_{w}=138.9083\right.$, $\mathrm{p}$-value $\left.=1.505059 \times 10^{-28}\right)$.

\section{Welch's heteroscedastic $F$ test with trimmed means and Winsorized variances: welch.test(...)}

The welch. test function in the onewaytests package is also used to perform Welch's heteroscedastic $F$ test with trimmed means and Winsorized variances.

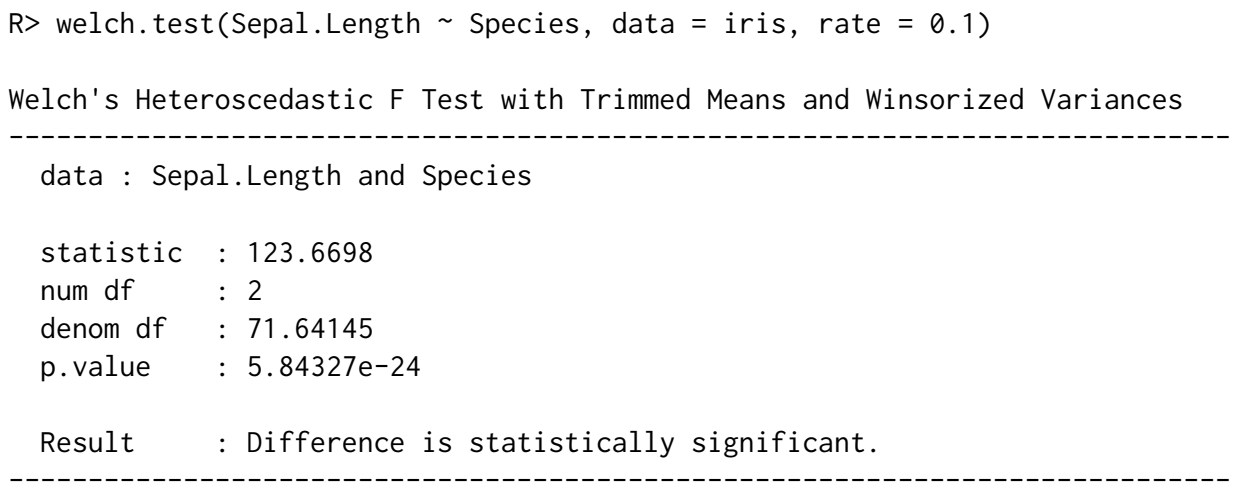

Here, the statistic is the test statistic distributed as F with the degrees of freedom for the numerator (num df) and denominator (denom df). Moreover, the rate is the rate of observations trimmed and Winsorized from each tail of the distribution. If rate $=0$, it performs Welch's heteroscedastic $F$ test. Otherwise, one can perform Welch's heteroscedastic $F$ test with trimmed means and Winsorized variances. Default is set to rate $=0$. One can conclude that there is a statistically significant difference between the species $\left(\mathrm{F}_{w t}=123.6698, \mathrm{p}\right.$-value $\left.=5.84327 \times 10^{-24}\right)$.

\section{Brown-Forsythe test: bf.test(...)}

One may use the bf. test function in the onewaytests package to perform Brown-Forsythe test to compare more than two groups.

$\mathrm{R}>$ bf.test(Sepal.Length $\sim$ Species, data $=$ iris)

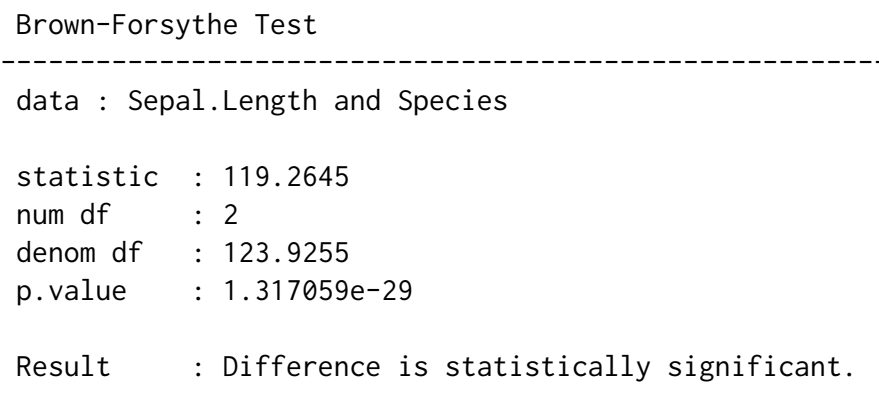

In the output, the statistic is the Brown-Forsythe test statistic distributed as F with the degrees of freedom for numerator (num df) and denominator (denom df). It can be concluded that there is a statistically significant difference between the iris species $\left(\mathrm{F}_{B F}=119.2645\right.$, $\mathrm{p}$-value $\left.=1.317059 \times 10^{-29}\right)$.

\section{Alexander-Govern test: ag.test(...)}

The ag. test function in the onewaytests package is used to perform Alexander-Govern test. 
R> ag.test (Sepal.Length $\sim$ Species, data = iris)

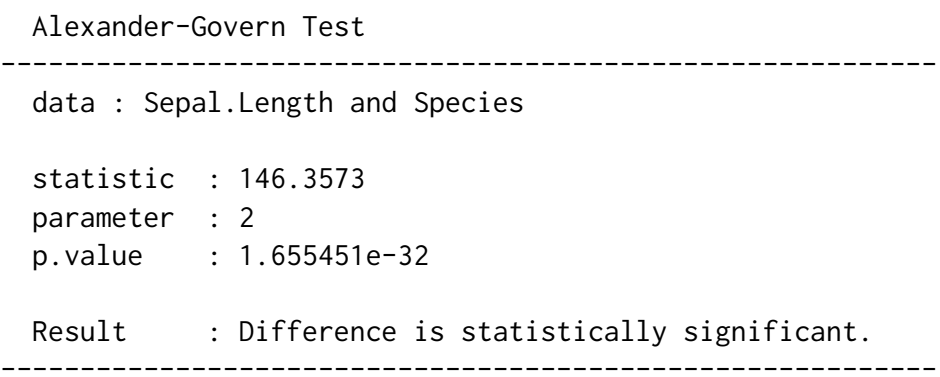

Here, statistic is the Alexander-Govern test statistic distributed as $\chi^{2}$ with the degrees of freedom (parameter). One can conclude that the difference between the species is statistically significant $\left(\chi_{A G}^{2}\right.$ $=146.3573$, p-value $=1.655451 \times 10^{-32}$ ).

\section{James second order test: james.test(...)}

One may use the james. test function in the onewaytests package to perform James second order test.

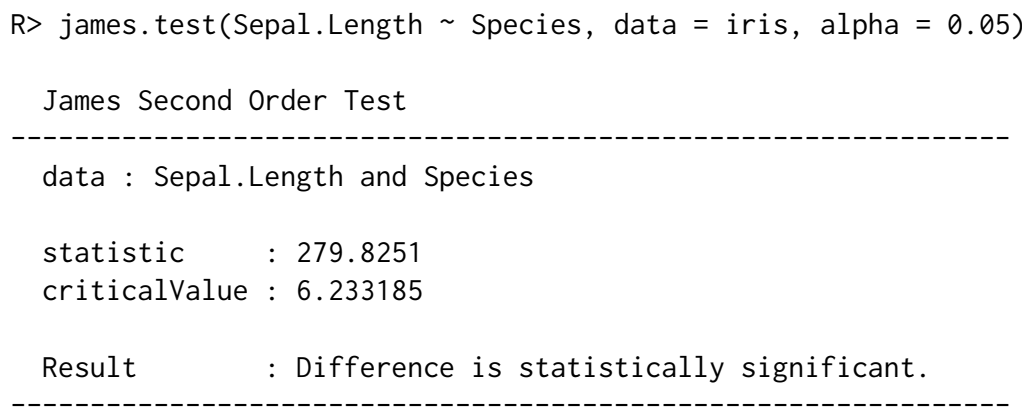

Here, alpha is the significance level, statistic is the James second order test statistic, J, criticalvalue is the critical value, $h(\alpha)$, corresponding to the significance level, $\alpha$. If $J$ exceeds $h(\alpha)$, then the null hypothesis is rejected. Since $J=279.8251$, obtained from james. test, is higher than $h(\alpha)=6.233185$, it can be concluded that there is a statistically significant difference between the iris species.

\section{Kruskal-Wallis test: kw.test(...)}

The kw. test function in the onewaytests package is utilized to perform Kruskal-Wallis test.

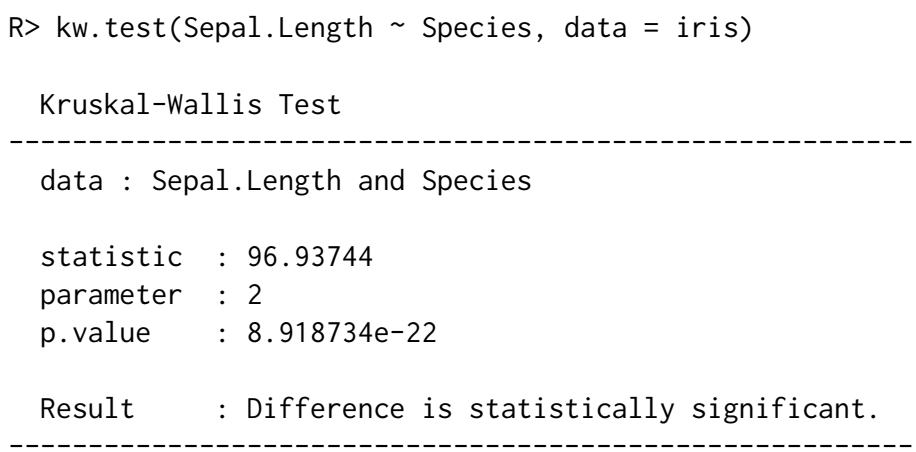

In the output, statistic is the Kruskal-Wallis test statistic distributed as $\chi^{2}$ with the degrees of freedom (parameter). One can conclude that the difference between the species is statistically significant $\left(\chi_{K W}^{2}=96.93744, \mathrm{p}\right.$-value $\left.=8.918734 \times 10^{-22}\right)$.

\section{Pairwise comparisons}

In this part, we present the pairwise comparisons to investigate which groups create the difference. We utilized the p.adjust function under the stats package (R Core Team, 2017) for our paircomp 
function. The paircomp function has also the same $p$-value adjustment methods as $p$.adjust, including bonferroni, holm (Holm, 1979), hochberg (Hochberg, 1988), hommel (Hommel, 1988), BH (Benjamini and Hochberg, 1995), BY (Benjamini and Yekutieli, 2001), and none. The default is set to "bonferroni". Pairwise comparisons are made by adjusting $p$-values according to the specified method, except when the method is James second order test, which requires adjusting the significance level instead of the p-value.

One-way tests return a list with class "owt" except for James second order test. The reason for returning a list with class "owt" is to introduce the output to the paircomp function for pairwise comparison adjusting p-values according to the specified method. Besides, James second order test returns a list with class "jt" introducing the output to the paircomp function for pairwise comparison by adjusting the significance level instead of the p-value.

In the iris example, there is a statistically significant difference between iris species in terms of sepal length measurements. All pairwise comparisons of groups can be conducted using paircomp function. For simplicity, the Bonferroni correction is applied to show the usage of the pairwise comparisons following the significant result obtained by Alexander-Govern test and James second order test.

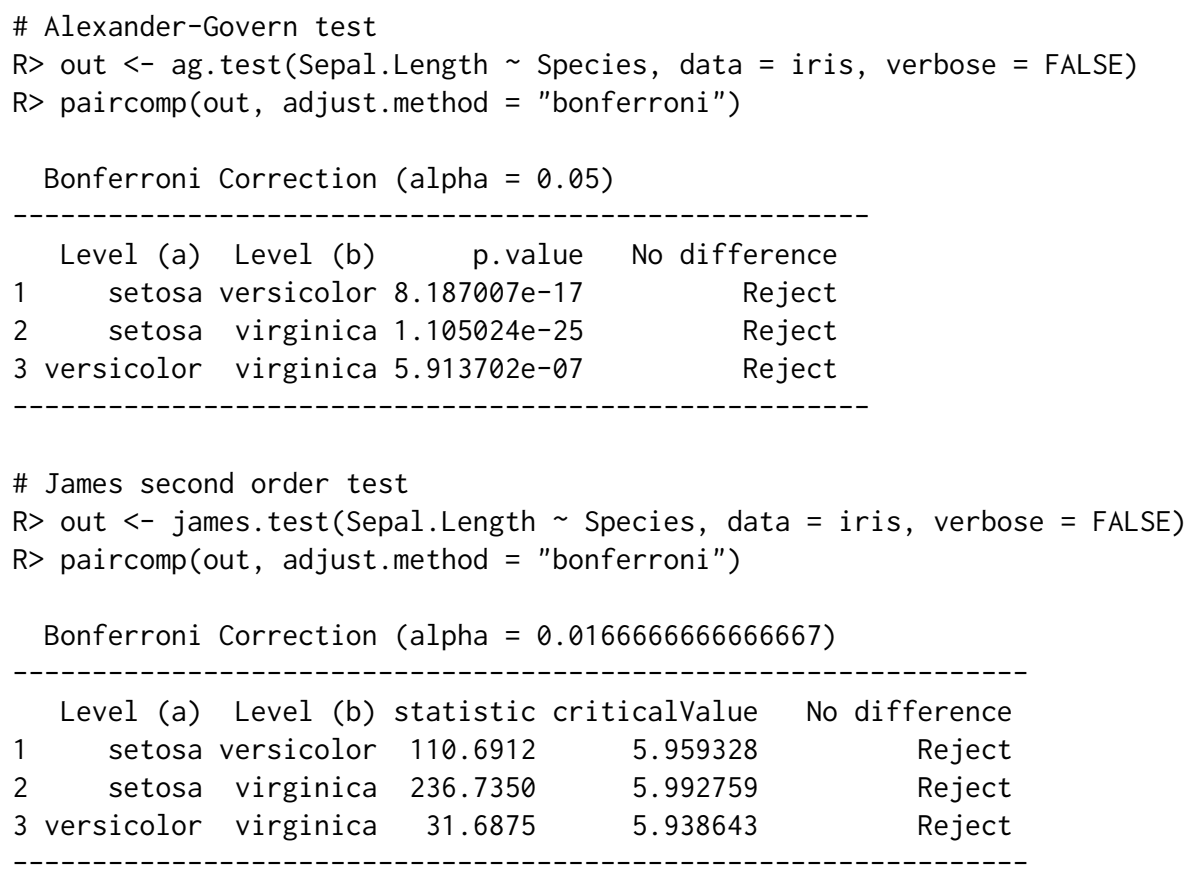

\begin{tabular}{|c|c|c|c|c|c|}
\hline & Level (a) & Level (b) & statistic & criticalvalue & No difference \\
\hline 1 & setosa & versicolor & 110.6912 & 5.959328 & Reject \\
\hline 2 & setosa & virginica & 236.7350 & 5.992759 & Reject \\
\hline 3 & versicolor & virginica & 31.6875 & 5.938643 & Reject \\
\hline
\end{tabular}

In both Alexander-Govern and James second order tests with Bonferroni correction, statistical differences between all types of iris flowers are significant in terms of sepal length measurements.

\section{Checking assumptions via tests and plots}

Two main assumptions, normality and variance homogeneity, can be checked through the onewaytests package. One can assess the variance homogeneity via homog. test, which has options including Levene (Levene's test), Bartlett (Bartlett's test) and Fligner (Fligner-Killeen test). Also, the normality of data in each group can be checked through nor. test, which has options including SW (Shapiro-Wilk test), SF (Shapiro-Francia test), LT (Lilliefors test known as Kolmogorov-Smirnov test), AD (AndersonDarling test), CVM (Cramer-von Mises test), PT (Pearson Chi-square test). Moreover, the nor . test has options to assess the normality of data in each group through plots (Q-Q plots and Histograms with normal curves).

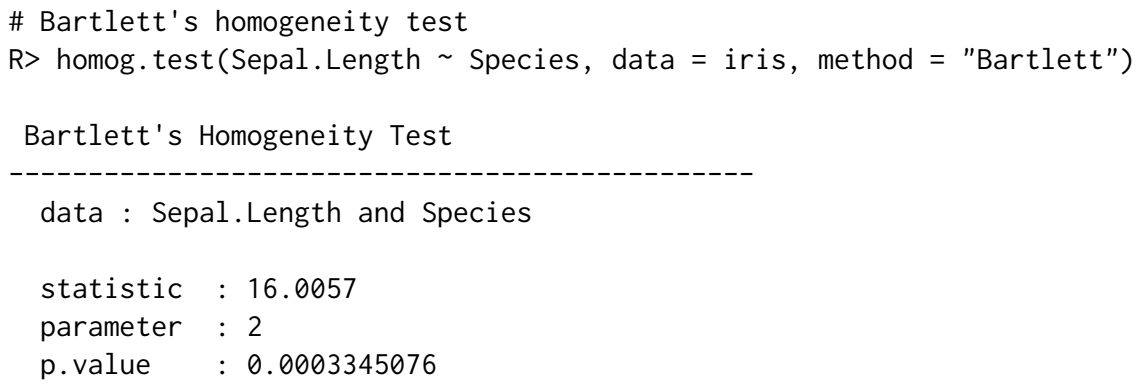


Result : Variances are not homogeneous.

Bartlett's homogeneity test results reveal that the variances between iris species are not homogeneous $\left(\chi^{2}=16.0057\right.$, $\mathrm{p}$-value $\left.=0.0003345076\right)$.

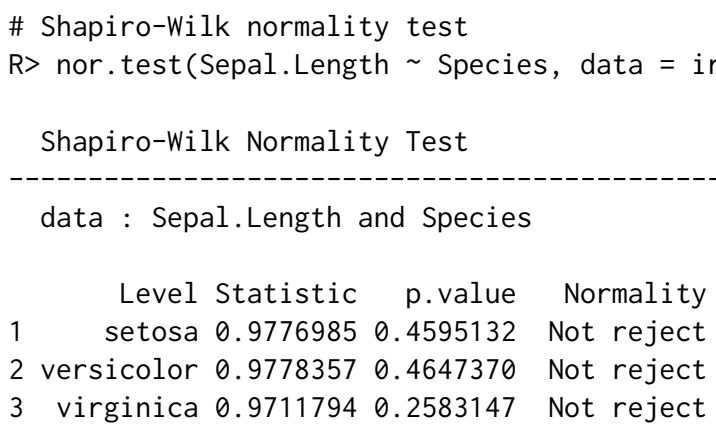

Shapiro-Wilk normality test results state that there is not enough evidence to reject the normality of sepal length values in each iris species since all $\mathrm{p}$-values are greater than 0.05 . Also, the normality of data in each group can be assessed visually by $\mathrm{Q}-\mathrm{Q}$ plots and histograms with normal curves (Figure 1).

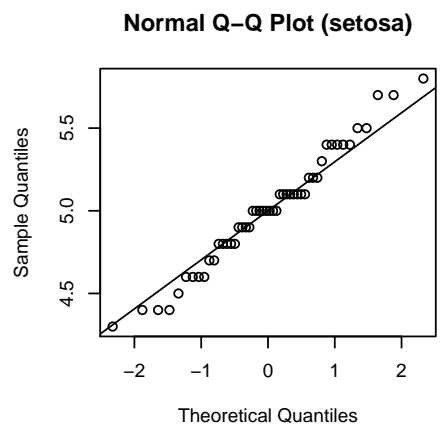

Histogram of setosa

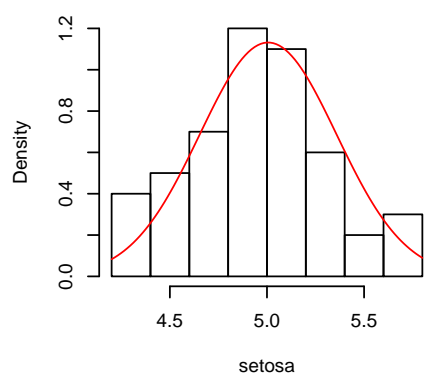

Normal Q-Q Plot (versicolor)

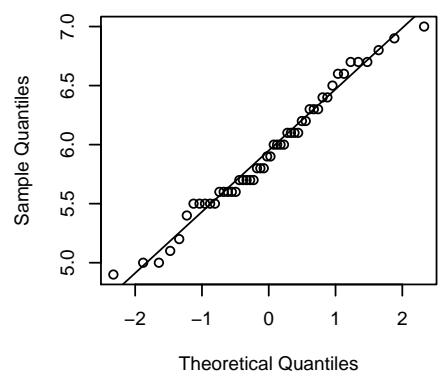

Histogram of versicolor

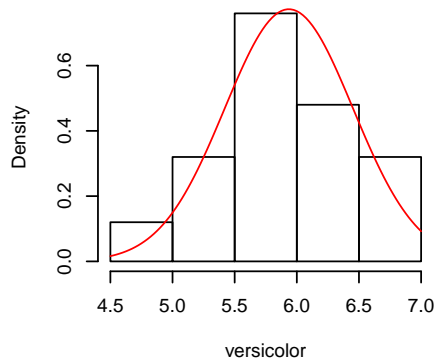

Normal Q-Q Plot (virginica)

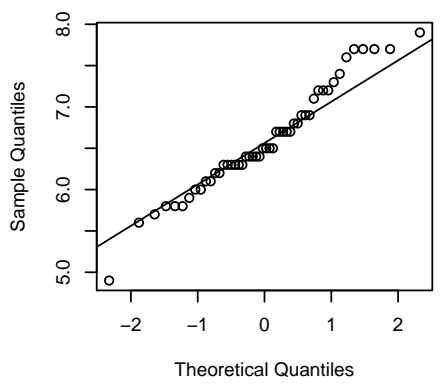

Histogram of virginica

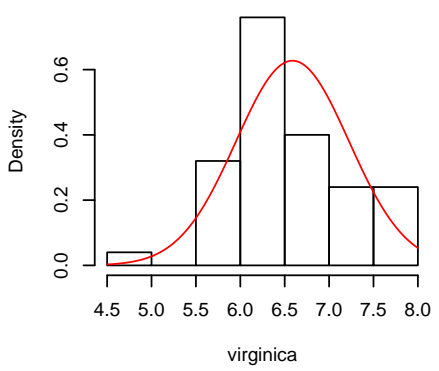

Figure 1: Q-Q plots and histograms with normal curves

\section{Graphical approaches}

The users can obtain several graphic types of given groups via the gplot, which has options involving box-and-whisker plot with violin line - a rotated density line on each side - (Figure 2a), box-andwhisker plot (Figure 2b), mean \pm standard deviation graph (Figure 2c) and mean \pm standard error graph (Figure 2d). These graphics can be obtained via the following codes:

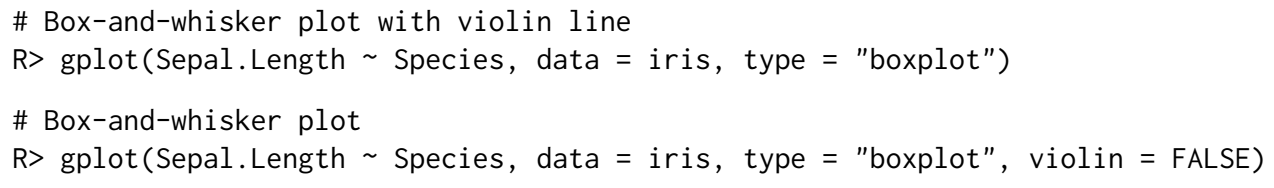


\# Mean +- standard deviation graph

R> gplot(Sepal.Length Species, data = iris, type = "errorbar", option = "sd")

\# Mean +- standard error graph

$\mathrm{R}>\operatorname{gplot}($ Sepal.Length $\sim$ Species, data $=$ iris, type $=$ "errorbar", option $=$ "se")

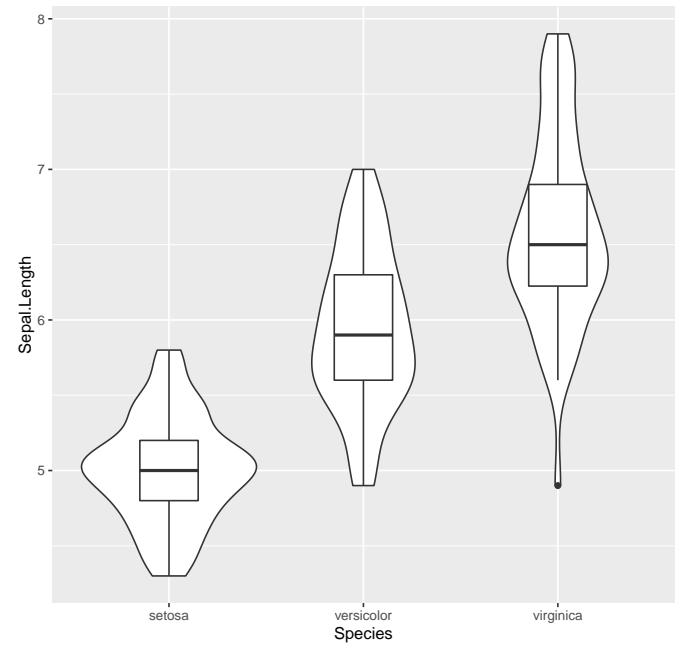

(a) Box-and-whisker plot with violin line

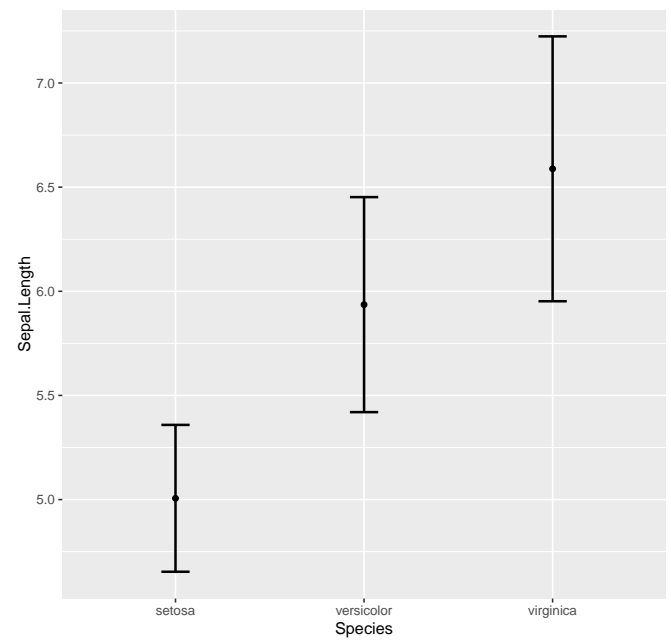

(c) Mean \pm standard deviation graph

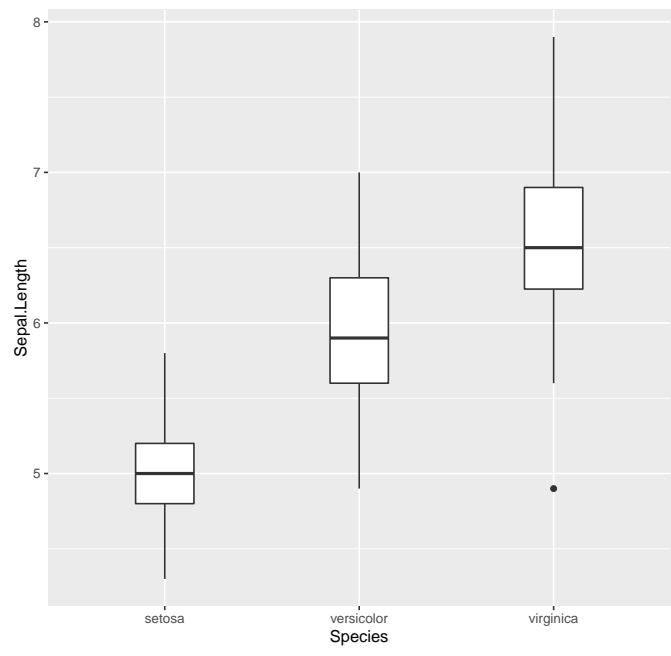

(b) Box-and-whisker plot

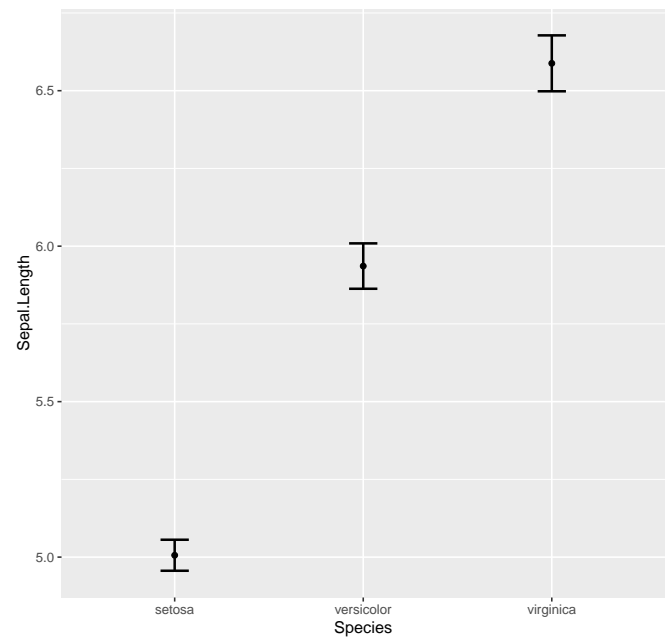

(d) Mean \pm standard error graph

Figure 2: Graphics of given groups

\section{German breast cancer data}

In this part, we utilize German breast cancer study group (GBSG) data set, available in the mfp package (Ambler and Benner, 2015) in R. This data set was collected from a cohort study and measurements from patients with primary node positive breast cancer were taken between July 1984 and December 1989. Seven risk factors, including menopausal status, tumor size, tumor grade, age, number of positive lymph nodes, progesterone and oestrogen receptor concentrations were examined in the work done by Sauerbrei et al. (1999). For illustrative purposes, we take the dependent variable as recurrence - free survival times of patients, of whom an event for recurrence-free survival occurs, and the factor as tumor grade. This factor is a three-level categorical variable: $1=$ grade I, 2 = grade II , and $3=$ grade III. A total of 299 observations (18, 202, 79 observations in each group, respectively) are available.

The objective of adding GBSG dataset is showing the usage of the package in practice rather than demonstrating the usage of all functions in the onewaytests package. After checking the normality and variance homogeneity assumptions, an appropriate one-way test is decided to compare groups. 
Also, pairwise comparisons are applied when it is necessary to determine which groups create the difference. $R$ code.

After installing and loading the mfp package, GBSG dataset can be reached by using the following

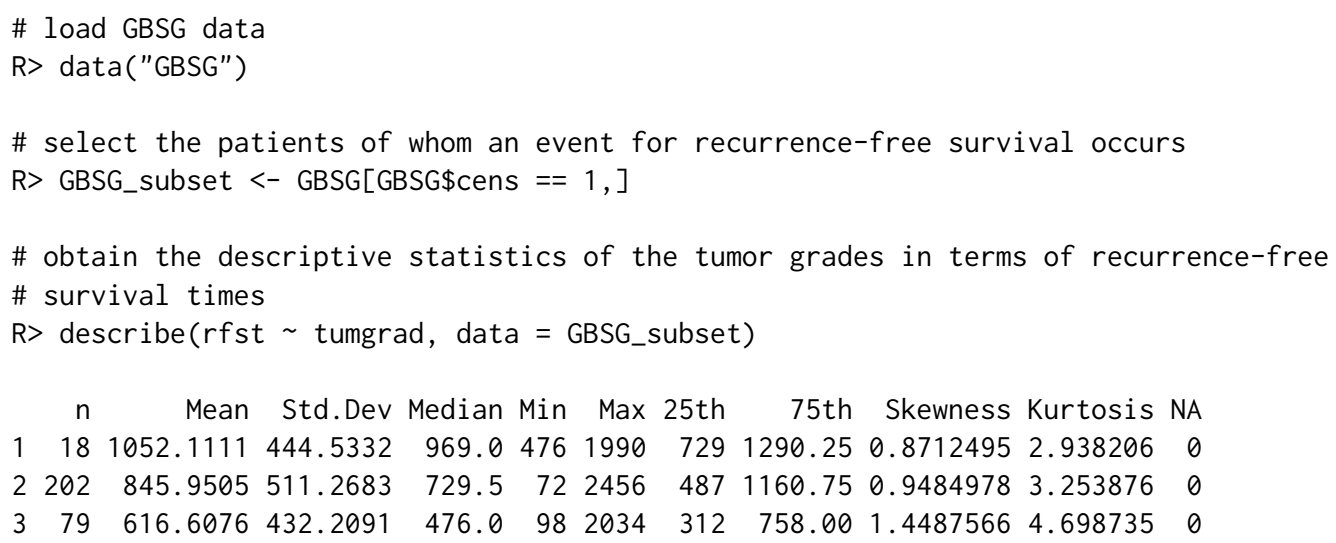

The assumptions of variance homogeneity and normality are assessed by using homog. test and nor . test, respectively.

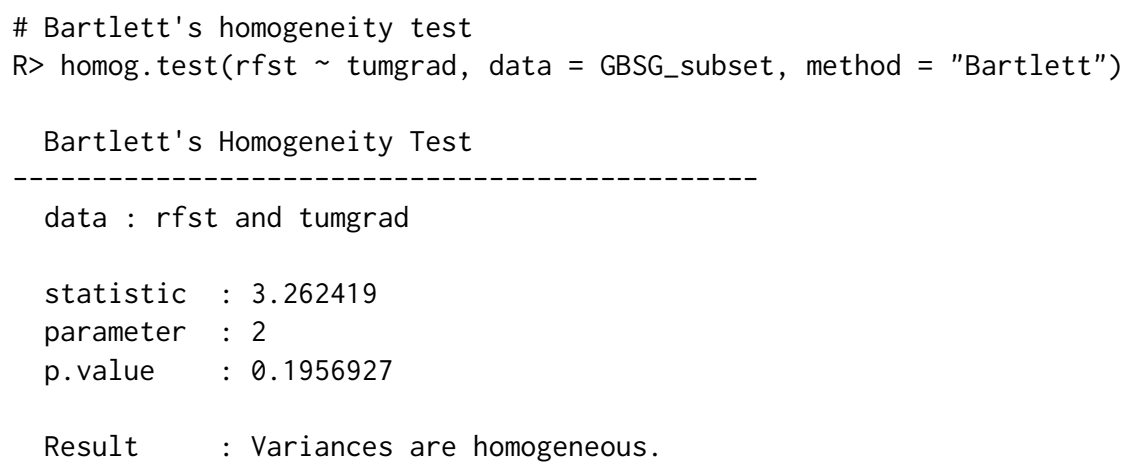

Bartlett's homogeneity test results reveal that there is no enough evidence to reject the variance homogeneity $\left(\chi^{2}=3.262419\right.$, $\mathrm{p}$-value $\left.=0.1956927\right)$ since $\mathrm{p}$-value is larger than 0.05 .

\# Shapiro-Wilk normality test

$\mathrm{R}>$ nor. test $($ rfst $\sim$ tumgrad, data $=$ GBSG_subset, method $=$ "SW")

Shapiro-Wilk Normality Test

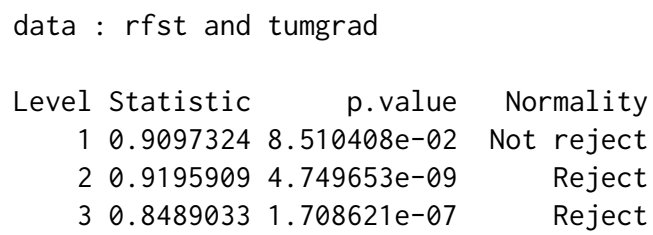

Shapiro-Wilk normality test results state that there is not enough evidence to reject the normality of recurrence - free survival times of the patients with tumor grade I since p-value is greater than 0.05 . The normality of recurrence - free survival times of the patients with tumor grade II and III is not met since the p-value is smaller than 0.05. Our simulation study results suggest that ANOVA is the appropriate one-way test in such case.

$\mathrm{R}>$ aov.test (rfst $\sim$ tumgrad, data $=$ GBSG_subset)

One-Way Analysis of Variance

data : rfst and tumgrad 


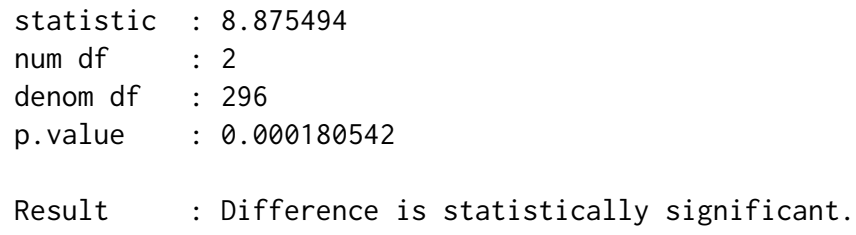

Since the p-value, derived from aov. test, is lower than 0.05 , it can be concluded that there is a statistically significant difference between the tumor grades with respect to recurrence - free survival times $(\mathrm{F}=8.875494$, $\mathrm{p}$-value $=0.000180542)$.

On the other hand, we observe that recurrence - free survival times of the patients with tumor grade II and III have few outliers and positively-skewed distributed. Liao et al. (2016) suggests that Kruskal-Wallis test should be used in such cases. Therefore, this test is also utilized along with ANOVA to compare groups.

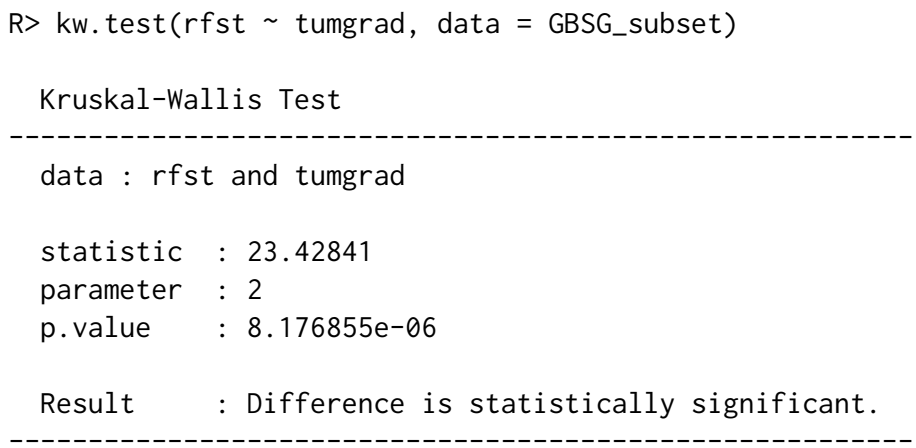

One can conclude that the difference between the tumor grades with respect to recurrence - free survival times is statistically significant $\left(\chi_{K W}^{2}=23.42841\right.$, $\mathrm{p}$-value $\left.=8.176855 \times 10^{-06}\right)$ since $\mathrm{p}$-value is smaller than 0.05 .

Both ANOVA and Kruskal-Wallis test results reveal that there is a statistically significant difference between tumor grade groups in terms of recurrence-free survival times. In the next step, we need to determine which groups create the difference.

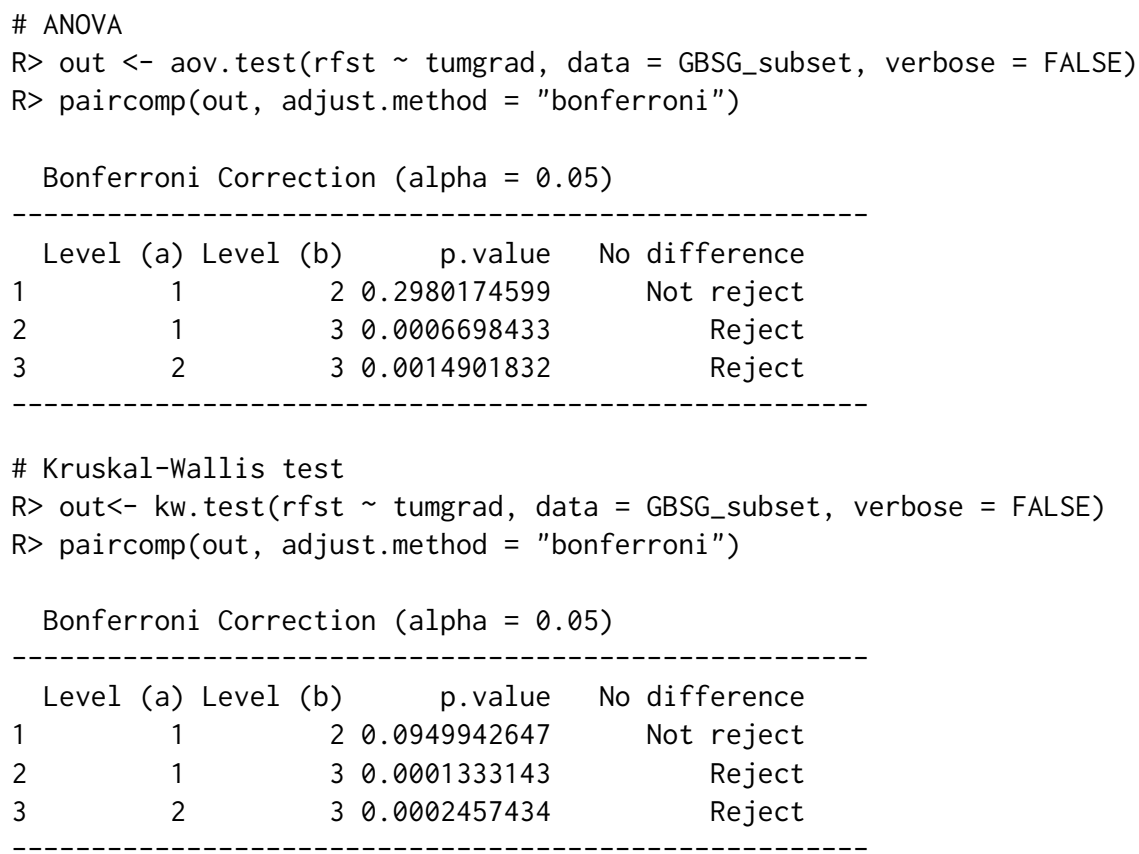

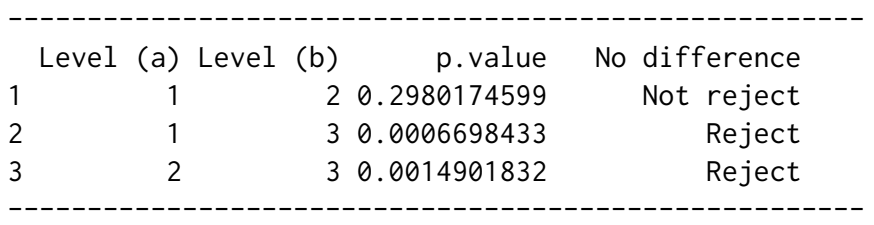

\# Kruskal-Wallis test

$\mathrm{R}>$ out $<-$ kw. test (rfst $\sim$ tumgrad, data $=$ GBSG_subset, verbose $=$ FALSE)

$\mathrm{R}>$ paircomp (out, adjust. method = "bonferroni")

Bonferroni Correction (alpha $=0.05)$

$\begin{array}{rrrrr} & \text { Level (a) Level (b) } & \text { p.value } & \text { No difference } \\ 1 & 1 & 2 & 0.0949942647 & \text { Not reject } \\ 2 & 1 & 3 & 0.0001333143 & \text { Reject } \\ 3 & 2 & 3 & 0.0002457434 & \text { Reject }\end{array}$

Pairwise comparisons with Bonferroni correction after both ANOVA and Kruskal-Wallis test indicate that there is no statistically significant difference between the patients with tumor grade I and 
those with tumor grade II in terms of recurrence-free survival times. On the other hand, there exists a statistically significant difference between the patients with tumor grade I/II and those with tumor grade III.

\section{Web interface of onewaytests package}

The objective of this package is to provide the users with one-way tests in independent groups designs, pairwise comparisons, graphical approaches, and assess variance homogeneity and normality of data in each group via tests and plots. At times, it is difficult for new $\mathrm{R}$ users or applied researchers to deal with $\mathrm{R}$ codes. Thus, we have developed a web interface of onewaytests package by using shiny (Chang et al., 2017). The web interface is available at http://www. softmed. hacettepe. edu. tr/onewaytests.

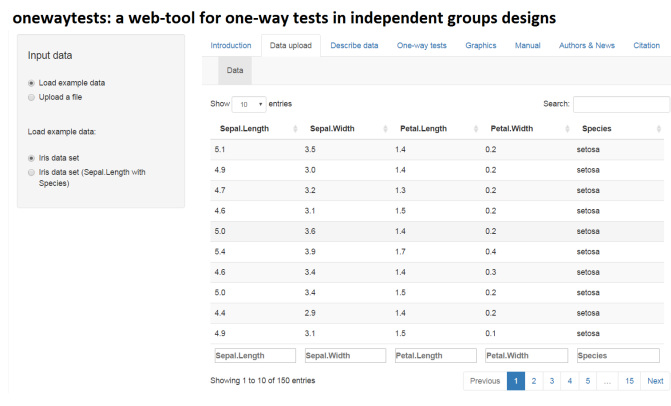

(a) Data upload

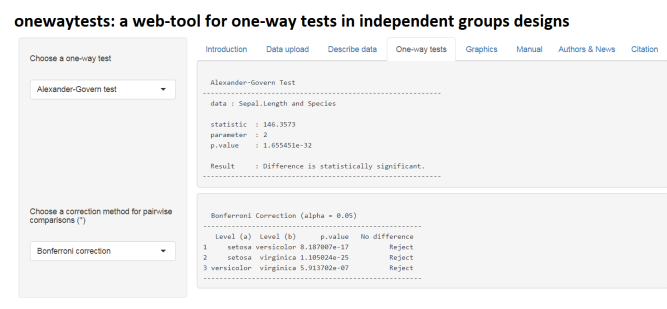

(c) One-way tests

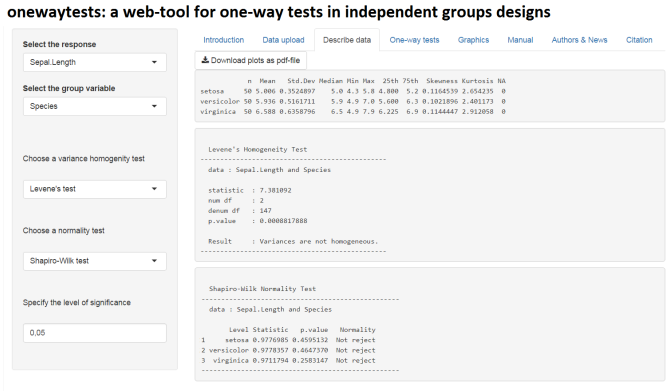

(b) Describe data

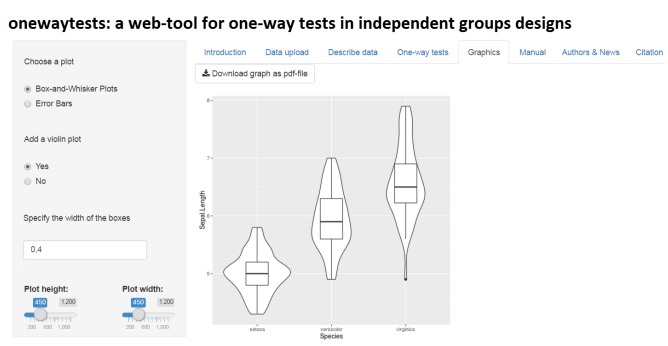

(d) Graphics

Figure 3: Web interface of onewaytests package

Users can upload their data to the tool via Data upload tab (Figure 3a). There exist two demo datasets on this tab for the users to test the tool. Basic descriptive statistics can be obtained through Describe data tab (Figure 3b). Moreover, assumptions of variance homogeneity and normality can be checked via this tab to decide on which one-way test is appropriate to test the statistical difference. Variance homogeneity is checked by variance homogeneity tests (Levene's test, Bartlett's test, FlignerKilleen test). The normality of data in each group is assessed by normality tests (Shapiro-Wilk, Cramer-von Mises, Lilliefors (Kolmogorov-Smirnov), Shapiro-Francia, Anderson-Darling, Pearson Chi-Square tests) and plots (Q-Q plot and Histogram with a normal curve). After describing the data, users can test statistical difference between groups through One-way tests tab (Figure 3c). In this tab, there exist one-way tests (ANOVA, Welch's heteroscedastic $F$, Welch's heteroscedastic $F$ test with trimmed means and Winsorized variances, Brown-Forsythe, Alexander-Govern, James second order test, Kruskal-Wallis tests) and pairwise comparison methods (Bonferroni, Holm, Hochberg, Hommel, Benjamini-Hochberg, Benjamini-Yekutieli, no corrections). Moreover, users can obtain the graphics of given groups (Figure 2) through Graphics tab (Figure 3d).

\section{Simulation study}

In this section, it is aimed to compare the performances of seven tests in terms of type I error and adjusted power (Lloyd, 2005), and give some general suggestions on which test(s) should be used or avoided under the violations of assumptions. The adjusted power, $R(\alpha)$, is

$$
R(\alpha)=\Phi\left(\hat{\delta}+\Phi^{-1}(\alpha)\right)
$$

with 


$$
\hat{\delta}=\Phi^{-1}(1-\hat{\beta})-\Phi^{-1}(\hat{\alpha}) .
$$

In Equation (10), $\hat{\alpha}$ is the estimated type I error, $\hat{\beta}$ is the estimated type II error, $\alpha$ is the nominal size for type I error, $\Phi$ and $\Phi^{-1}$ are the cumulative and inverse cumulative distribution functions of the standard normal distribution, respectively.

\section{Simulation design}

A Monte Carlo simulation is implemented to illustrate the performances of these tests for the scenarios in which the assumptions of normality and/or variance homogeneity are held or not. The algorithm of the simulation study can be depicted as follows:

i) Generate three random samples from normal or skew normal distribution with means $\mu_{1}, \mu_{2}$ and $\mu_{3}$ and standard deviations $\sigma_{1}, \sigma_{2}, \sigma_{3}$. Group standard deviations are taken as $\sigma_{1}=\sigma_{2}=\sigma_{3}=1$ for homogeneous case, and $\sigma_{1}=1, \sigma_{2}=\sqrt{2}, \sigma_{3}=2$ for heterogeneous case. Skewness $\gamma$ is set to $\gamma=0$ for normal distribution, $\gamma=0.5$ for positive skew normal distribution and $\gamma=-0.5$ for negative skew normal distribution with different sample size combinations (balanced and unbalanced). Set $\mu_{1}=\mu_{2}=\mu_{3}=0$ for gathering type I error; $\mu_{1}=0, \mu_{2}=0.25, \mu_{3}=0.5$ or $\mu_{1}=0, \mu_{2}=0.5, \mu_{3}=1$ or $\mu_{1}=0, \mu_{2}=1, \mu_{3}=2$ for power.

ii) Check whether groups are different by the corresponding one-way test in independent groups designs at the level of significance $\alpha(\alpha=0.05)$.

iii) Repeat steps i) - ii) for 10,000 times and calculate the probability of rejecting the null hypothesis when the null hypothesis is true (type I error) or false (power).

iv) Calculate adjusted power (given in Lloyd (2005)) by using the estimated type I error and power found in iii).

\section{Results}

In this section, the performances of one-way tests in independent groups designs are investigated through type I error and adjusted power. All results are not given here to protect the content integrity, but attached as supplementary tables $2-4$.

\section{Type I error rates}

In this part, we compare seven one-way tests in terms of their type I errors. We observed that the type I errors get closer to nominal level as sample size increases under the data generated from normal distribution $(\gamma=0)$ with equal variances $\left(\sigma_{1}=\sigma_{2}=\sigma_{3}=1\right)$. Kruskal-Wallis test tends to be more conservative compared to the rest of them in the unbalanced small sample sizes $\left(n_{1}=6, n_{2}=9, n_{3}=\right.$ $15)$. When variance homogeneity is not held under normality, unbalance of sample sizes causes a decline in type I error rates of ANOVA and Kruskal-Wallis test regardless of sample size.

When the data in each group come from right skew normal distribution $(\gamma=0.5)$ with unbalanced small sample size setting, type I error rate of ANOVA is estimated to be 0.052 under variance homogeneity $\left(\sigma_{1}=\sigma_{2}=\sigma_{3}=1\right)$ whereas it declines to 0.026 under variance heterogeneity $\left(\sigma_{1}=1, \sigma_{2}=\sqrt{2}, \sigma_{3}=2\right)$. The type I error rate of ANOVA is negatively affected by the combined effect of heterocedasticity and unbalanced sample size. Especially, when the smaller variances are associated with groups having smaller sample size, the empirical type I error rate of ANOVA is halved compared to the homoscedastic case. Kruskal-Wallis test is dramatically affected by variance heterogeneity. It tends to be conservative in the unbalanced case whereas it becomes liberal in the balanced case.

James second order test, Alexander-Govern test, Welch's heteroscedastic $F$ test and Welch's heteroscedastic $F$ test with trimmed means and Winsorized variances control the type I error rate at the nominal size for all simulation scenarios. Brown-Forsythe test is not able to control type I error rate; especially when the variances are heterogeneous, it becomes liberal.

\section{Adjusted powers}

Adjusted power is important to compare tests having different type I errors since it adjusts power with respect to type I error. The results are illustrated in Figures $4-5$ to see the clear difference among the tests.

Within the tests discussed in this study, ANOVA is the best one with respect to adjusted power when the sample sizes are not equal under normality $(\gamma=0)$ and variance homogeneity $\left(\sigma_{1}=\sigma_{2}=\right.$ 
$\sigma_{3}=1$ ). Under the same condition, ANOVA and Brown-Forsythe test are superior to the rest of them in terms of adjusted power when the design of sample size is balanced. As expected, ANOVA performs poor under normality when variances are unstable $\left(\sigma_{1}=1, \sigma_{2}=\sqrt{2}, \sigma_{3}=2\right)$; however, Alexander-Govern, James and Welch's heteroscedastic $F$ tests have higher adjusted powers compared to other tests. Brown-Forsythe and Kruskal-Wallis tests perform poorly compared to the others under the same condition.

When the data are generated from positive skew normal distribution $(\gamma=0.5)$ with equal variances, ANOVA and Kruskal-Wallis test perform better than other tests. ANOVA is slightly better than Kruskal-Wallis test for small sample sizes and vice versa is true for the medium sample sizes. James, Alexander-Govern and Welch's heteroscedastic $F$ tests have the highest adjusted powers among the whole tests for all scenario combinations under the data generated from a positive skew normal distribution with heterogeneous variances.

ANOVA, Brown-Forsythe test and Kruskal-Wallis test perform better than the other tests when the data are generated from negative skew normal distribution $(\gamma=-0.5)$ with homogeneous variances and equal sample sizes; however, Kruskal-Wallis test is slightly superior to ANOVA and BrownForsythe test. The adjusted power of ANOVA seems not to be affected by the skewness and it performs best under the same condition when the design of sample size is unbalanced. When the data are generated from the negative skew normal distribution with heterogeneous variances, Kruskal-Wallis test performs best for low $\left(\mu_{1}=0, \mu_{2}=0.25, \mu_{3}=0.5\right)$ and medium $\left(\mu_{1}=0, \mu_{2}=0.5, \mu_{3}=1\right)$ effect sizes while Welch's heteroscedastic $F$ test with trimmed mean and Winsorized variance has higher performance compared to other tests for high effect size $\left(\mu_{1}=0, \mu_{2}=1, \mu_{3}=2\right)$.

It is noted that the Kruskal-Wallis test is affected by the skewness of the distribution especially under heterogeneity of variance $\left(\sigma_{1}=1, \sigma_{2}=\sqrt{2}, \sigma_{3}=2\right)$. Kruskal-Wallis test has the highest adjusted power when the data are generated from a negatively skewed distribution $(\gamma=-0.5)$. On the other hand, it has the lowest adjusted power when the data are generated from a positively skewed distribution $(\gamma=0.5)$. 


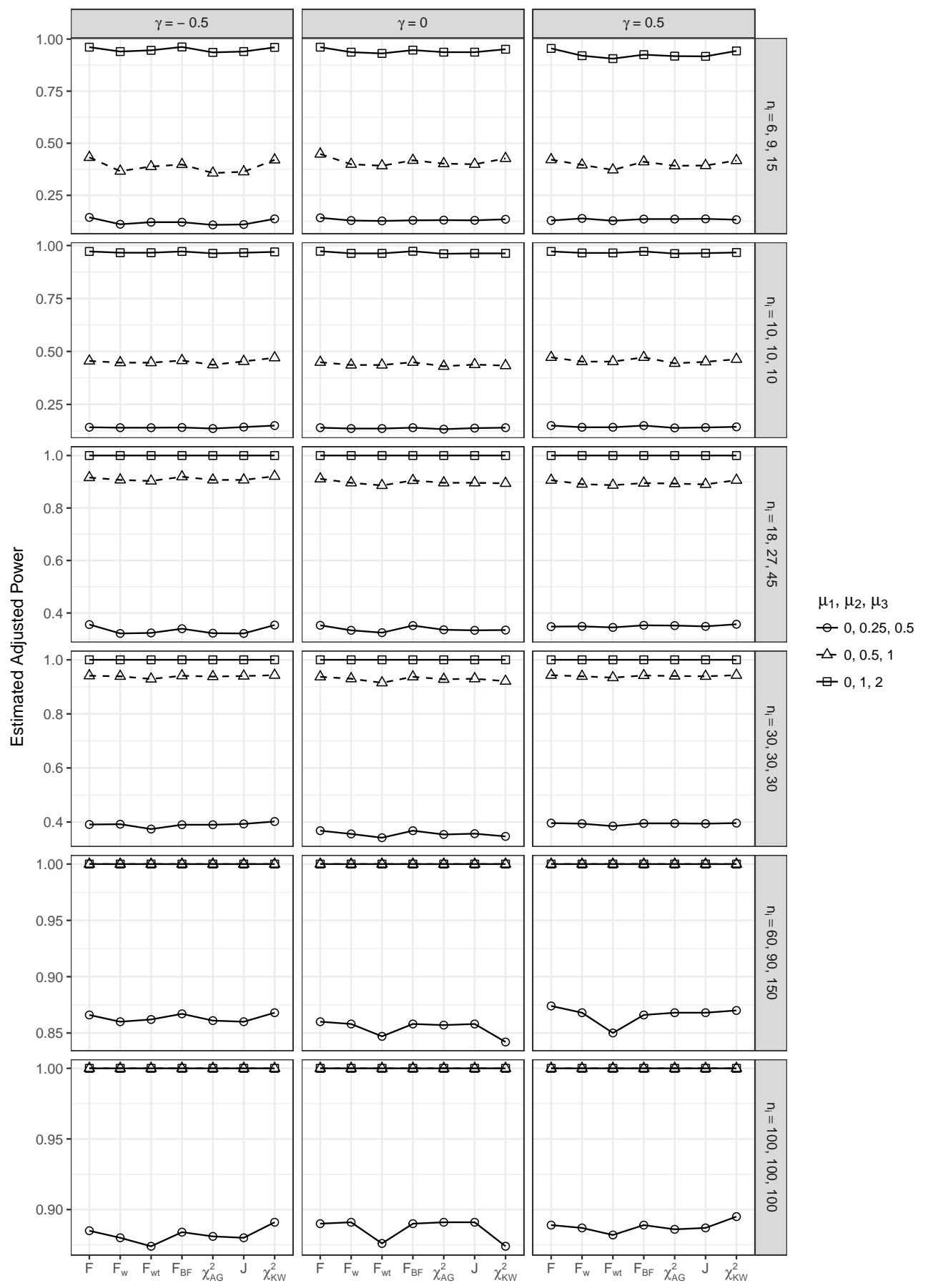

Figure 4: Estimated adjusted power results under homogeneous-variance $\left(\sigma_{1}=\sigma_{2}=\sigma_{3}=1\right)$ scenarios 


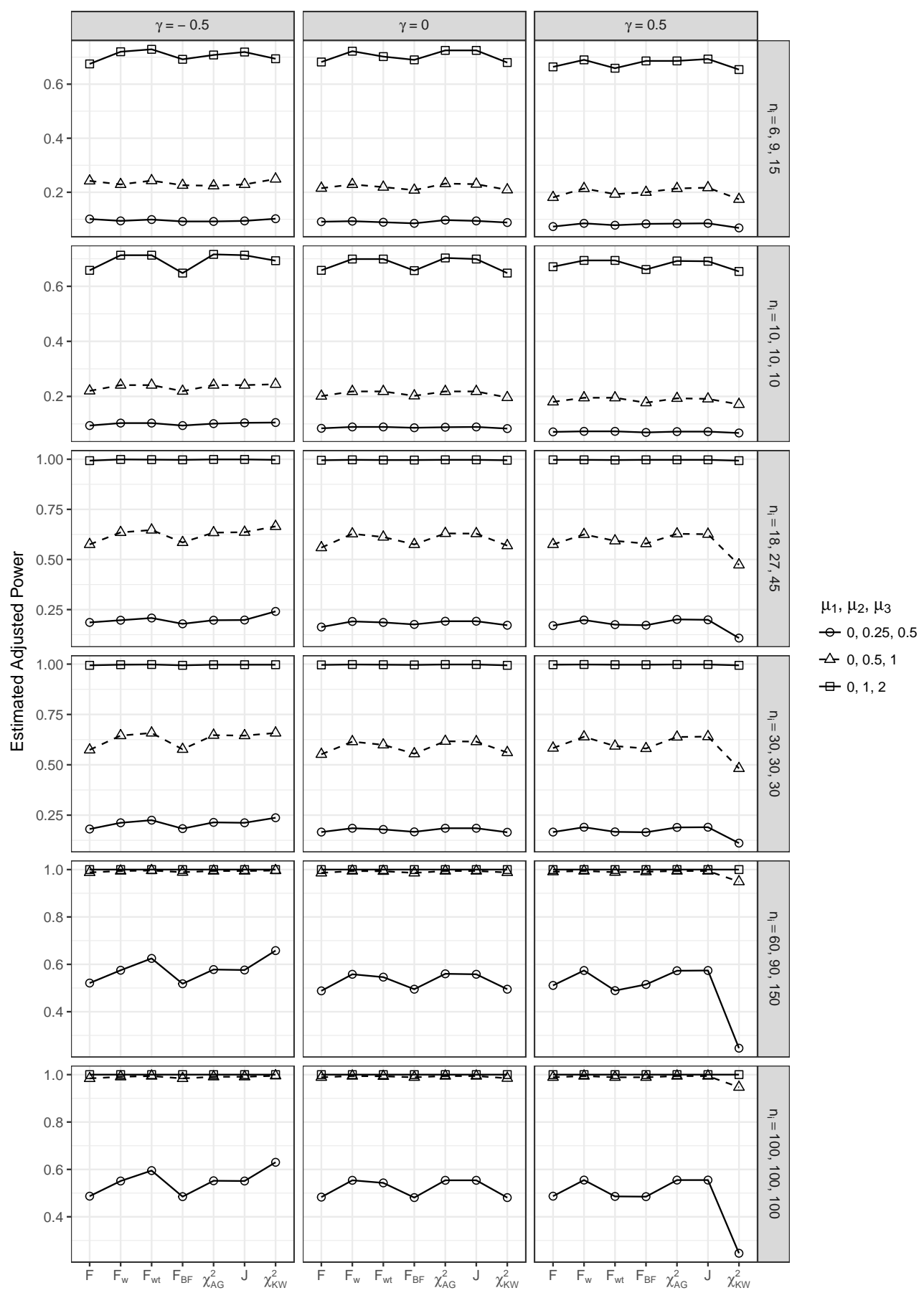

Figure 5: Estimated adjusted power results under heterogeneous-variance $\left(\sigma_{1}=1, \sigma_{2}=\sqrt{2}, \sigma_{3}=2\right)$ scenarios

\section{Summary and further research}

One-way tests in independent groups designs are the most commonly utilized statistical methods with applications on the experiments in medical sciences, pharmaceutical research, agriculture, biology, chemistry, engineering, and social sciences. In this paper, we present the onewaytests package for researchers to investigate treatment effects on the dependent variable.

The onewaytests package includes well-known one-way tests in independent groups designs including one-way analysis of variance, Kruskal-Wallis test, Welch heteroscedastic $F$ test, BrownForsythe test. In addition to these well-known tests, Alexander-Govern test, James second order test 
and Welch heteroscedastic $F$ test with trimmed means and Winsorized variances, not available in most statistical software, are able to be reached via this package. Also, pairwise comparisons can be applied if the statistically significant difference is obtained.

Normality and variance homogeneity are the vital assumptions for the selection of the appropriate test. Therefore, this package also enables the users to check these assumptions via variance homogeneity and normality tests. It also enables the users some basic descriptive statistics and graphical approaches.

ANOVA is the most commonly used method for one-way independent groups designs. However, this method has two certain assumptions, homogeneity of variances and normality, to be satisfied. When these assumptions are not met, there are some other alternatives for one-way independent groups designs, which include Welch's heteroscedastic $F$ test, Welch's heteroscedastic $F$ test with trimmed means and Winsorized variances, Alexander-Govern test, James second order test, BrownForsythe test and Kruskal-Wallis test.

In this paper, we also compared seven one-way tests in onewaytests package with respect to the type I error rate and adjusted power. In the light of Monte Carlo simulation, it is noted that the normality assumption does not have a negative effect on the adjusted power of the tests as severe as variance homogeneity assumption. ANOVA is suggested to be used if the variance homogeneity assumption is held. Otherwise, Alexander-Govern, James second order and Welch's heteroscedastic $F$ tests are recommended to be utilized. It is pointed out that under the negatively skew normal distribution with heterogeneous variances, Kruskal-Wallis test performs best with small and medium effect sizes while the Welch's heteroscedastic $F$ test with trimmed means and Winsorized variances has the highest adjusted power with large effect size.

At present, the onewaytests package offers the one-way tests in independent groups designs, pairwise comparisons, graphical approaches, and assessment of variance homogeneity and normality via tests and plots. The package and its web-interface will be updated at regular intervals.

\section{Acknowledgment}

We thank the anonymous reviewers for their constructive comments and suggestions which helped us to improve the quality of our paper.

\section{Bibliography}

R. A. Alexander and D. M. Govern. A New and Simpler Approximation for ANOVA under Variance Heterogeneity. Journal of Educational and Behavioral Statistics, 19(2):91-101, 1994. URL https: //doi.org/10.2307/1165140. [p175, 176, 178, 179]

G. Ambler and A. Benner. Mfp: Multivariable Fractional Polynomials, 2015. R package version 1.5.2. [p186]

E. Anderson. The Irises of the Gaspe Peninsula. Bulletin of the American Iris society, 59:2-5, 1935. [p180]

Y. Benjamini and Y. Hochberg. Controlling the False Discovery Rate: a Practical and Powerful Approach to Multiple Testing. Journal of the Royal Statistical Society. Series B (Methodological), 57(1): 289-300, 1995. [p184]

Y. Benjamini and D. Yekutieli. The control of the false discovery rate in multiple testing under dependency. The Annals of Statistics, 29(4):1165-1188, 2001. URL https://doi .org/10.1214/aos/ 1013699998. [p184]

T. A. Bishop. Heteroscedastic ANOVA, MANOVA, and Multiple-Comparisons. PhD thesis, The Ohio State University, 1976. [p176]

M. B. Brown and A. B. Forsythe. The Small Sample Behavior of Some Statistics Which Test the Equality of Several Means. Technometrics, 16(1):129-132, 1974a. URL https://doi .org/10. 2307/1267501. [p175, 176, 178]

M. B. Brown and A. B. Forsythe. Robust Tests for the Equality of Variances. Journal of the American Statistical Association, 69(346):364-367, 1974b. [p175, 176, 178]

W. Chang, J. Cheng, J. Allaire, Y. Xie, and J. McPherson. shiny: Web Application Framework for R, 2017. R package version 1.0.1. [p189] 
R. A. Cribbie, R. R. Wilcox, C. Bewell, and H. Keselman. Tests for Treatment Group Equality When Data Are Nonnormal and Heteroscedastic. Journal of Modern Applied Statistical Methods, 6(1):117-132, 2007. URL https://doi .org/10.22237/jmasm/1177992660. [p175]

R. A. Cribbie, L. Fiksenbaum, H. Keselman, and R. R. Wilcox. Effect of Non-Normality on Test Statistics for One-Way Independent Groups Designs. British Journal of Mathematical and Statistical Psychology, 65(1):56-73, 2012. URL https://doi.org/10.1111/j.2044-8317.2011.02014.x. [p175]

O. Dag, A. Dolgun, and N. M. Konar. Onewaytests: One-Way Tests in Independent Groups Designs, 2017. R package version 1.5. [p175]

M. W. Fagerland and L. Sandvik. The Wilcoxon-Mann-Whitney Test under Scrutiny. Statistics in Medicine, 28(10):1487-1497, 2009. URL https://doi .org/10. 1002/sim. 3561. [p179]

R. A. Fisher. The Use of Multiple Measurements in Taxonomic Problems. Annals of Eugenics, 7(2): 179-188, 1936. URL https://doi.org/10.1111/j.1469-1809.1936.tb02137.x. [p180]

J. Fox and S. Weisberg. An R Companion to Applied Regression. Sage, Thousand Oaks CA, 2nd edition, 2011. [p179]

J. Gamage and S. Weerahandi. Size Performance of Some Tests in One-Way ANOVA. Communications in Statistics-Simulation and Computation, 27(3):625-640, 1998. URL https://doi .org/10.1080/ 03610919808813500. [p175]

J. L. Gastwirth, Y. R. Gel, W. L. W. Hui, V. Lyubchich, W. Miao, and K. Noguchi. lawstat: Tools for Biostatistics, Public Policy, and Law, 2017. R package version 3.1. [p179]

J. Gross and U. Ligges. Nortest: Tests for Normality, 2015. R package version 1.0-4. [p180]

Y. Hochberg. A Sharper Bonferroni Procedure for Multiple Tests of Significance. Biometrika, 75(4): 800-802, 1988. [p184]

S. Holm. A simple sequentially rejective multiple test procedure. Scandinavian Journal of Statistics, 6(2): 65-70, 1979. [p184]

G. Hommel. A Stagewise Rejective Multiple Test Procedure Based on a Modified Bonferroni Test. Biometrika, 75(2):383-386, 1988. [p184]

T. Hothorn, K. Hornik, M. A. Van De Wiel, and A. Zeileis. A Lego System for Conditional Inference. The American Statistician, 60(3):257-263, 2006. URL https://doi .org/10.1198/000313006X118430. [p179]

Y.-C. Hu. A new fuzzy-data mining method for pattern classification by principal component analysis. Cybernetics and Systems: An International Journal, 36(5):527-547, 2005. URL https://doi .org/10. 1080/01969720590944294. [p180]

G. S. James. The comparison of several groups of observations when the ratios of the population variances are unknown. Biometrika, 38(3/4):324-329, 1951. URL https://doi . org/10.2307/2332578. [p175, 176, 178]

G. S. James. Tests of linear hypotheses in univariate and multivariate analysis when the ratios of the population variances are unknown. Biometrika, 41(1-2):19-43, 1954. URL https://doi .org/10. 2307/2333003. [p175, 176]

H. Keselman, J. Algina, L. M. Lix, R. R. Wilcox, and K. N. Deering. A Generally Robust Approach for Testing Hypotheses and Setting Confidence Intervals for Effect Sizes. Psychological Methods, 13(2): 110-129, 2008. URL https://doi.org/10.1037/1082-989x.13.2.110. [p177]

S. Korkmaz, D. Goksuluk, and G. Zararsiz. MVN: An R Package for Assessing Multivariate Normality. The R Journal, 6(2):151-162, 2014. [p180]

W. H. Kruskal and W. A. Wallis. Use of Ranks in One-Criterion Variance Analysis. Journal of the American Statistical Association, 47(260):583-621, 1952. [p175, 176, 179]

B. Lantz. The Impact of Sample Non-Normality on ANOVA and Alternative Methods. British Journal of Mathematical and Statistical Psychology, 66(2):224-244, 2013. URL https://doi .org/10.1111/j .20448317.2012.02047.x. [p175, 176]

H. Liao, Y. Li, and G. Brooks. Outlier Impact and Accommodation Methods: Multiple Comparisons of Type I Error Rates. Journal of Modern Applied Statistical Methods, 15(1):23, 2016. URL https: //doi.org/10.22237/jmasm/1462076520. [p188] 
C. J. Lloyd. Estimating Test Power Adjusted for Size. Journal of Statistical Computation and Simulation, 75(11):921-933, 2005. URL https://doi .org/10.1080/00949650412331321160. [p189,190]

P. Mair, F. Schoenbrodt, and R. Wilcox. WRS2: Wilcox Robust Estimation and Testing, 2017. 0.9-2. [p179]

L. Myers. Comparability of the James' Second-Order Approximation Test and the Alexander and Govern A Statistic for Non-Normal Heteroscedastic Data. Journal of Statistical Computation and Simulation, 60(3):207-222, 1998. URL https://doi .org/10. 1080/00949659808811888. [p178]

T. Oshima and J. Algina. Type I Error Rates for James's Second-Order Test and Wilcox's Hm Test under Heteroscedasticity and Non-Normality. British Journal of Mathematical and Statistical Psychology, 45 (2):255-263, 1992. URL https://doi .org/10.1111/j.2044-8317.1992. tb00991.x. [p179]

I. Parra-Frutos. Testing Homogeneity of Variances with Unequal Sample Sizes. Computational Statistics, 28(3):1269-1297, 2013. URL https://doi .org/10.1007/s00180-012-0353-x. [p175]

R Core Team. R: a Language and Environment for Statistical Computing. R Foundation for Statistical Computing, Vienna, Austria, 2017. URL https://www.R-project.org/. [p179, 183]

W. Sauerbrei, P. Royston, H. Bojar, C. Schmoor, M. Schumacher, G. B. C. S. Group, and others. Modelling the effects of standard prognostic factors in node-positive breast cancer. British Journal of Cancer, 79: 1752-1760, 1999. URL https://doi.org/10.1038/sj.bjc.6690279. [p186]

P. J. Villacorta. The welchADF Package for Robust Hypothesis Testing in Unbalanced Multivariate Mixed Models with Heteroscedastic and Non-Normal Data. The R Journal, 2017. [p179]

B. L. Welch. On the comparison of several mean values - an alternative approach. Biometrika, 38(3/4): 330-336, 1951. URL https://doi.org/10.2307/2332579. [p175, 176, 177]

H. Wickham. Ggplot2: Elegant Graphics for Data Analysis. Springer-Verlag, 2009. [p180]

R. R. Wilcox. A New Alternative to the ANOVA F and New Results on James's Second-Order Method. British Journal of Mathematical and Statistical Psychology, 41(1):109-117, 1988. URL https: //doi.org/10.1111/j.2044-8317.1988. tb00890.x. [p175]

\author{
Osman Dag \\ Department of Biostatistics \\ Hacettepe University Faculty of Medicine, Sihhiye, Ankara \\ Turkey \\ osman. dag@hacettepe.edu. tr \\ Anil Dolgun \\ School of Science \\ RMIT University, Melbourne \\ Australia \\ anil.dolgun@rmit.edu.au \\ Naime Meric Konar \\ Department of Biostatistics \\ Hacettepe University Faculty of Medicine, Sihhiye, Ankara \\ Turkey \\ nmeric. konar@hacettepe.edu.tr
}




\section{Supplementary Material}

Table 2: Estimated type I error rates

\begin{tabular}{|c|c|c|c|c|c|c|c|c|c|}
\hline Distribution & $\sigma_{1}, \sigma_{2}, \sigma_{3}$ & $n_{1}, n_{2}, n_{3}$ & $F$ & $F_{w}$ & $F_{w t}$ & $F_{B F}$ & $\chi_{A G}^{2}$ & $J$ & $\chi_{K W}^{2}$ \\
\hline \multirow{12}{*}{$\begin{array}{l}\text { Normal } \\
(\gamma=0)\end{array}$} & \multirow{6}{*}{$1,1,1$} & $6,9,15$ & 0.046 & 0.047 & 0.047 & 0.046 & 0.046 & 0.047 & 0.041 \\
\hline & & $10,10,10$ & 0.052 & 0.050 & 0.050 & 0.050 & 0.049 & 0.051 & 0.047 \\
\hline & & $18,27,45$ & 0.049 & 0.051 & 0.051 & 0.048 & 0.051 & 0.051 & 0.048 \\
\hline & & $30,30,30$ & 0.051 & 0.052 & 0.053 & 0.051 & 0.052 & 0.052 & 0.051 \\
\hline & & $60,90,150$ & 0.050 & 0.049 & 0.050 & 0.051 & 0.049 & 0.049 & 0.050 \\
\hline & & $100,100,100$ & 0.051 & 0.050 & 0.052 & 0.051 & 0.050 & 0.050 & 0.050 \\
\hline & \multirow{6}{*}{$1, \sqrt{2}, 2$} & $6,9,15$ & 0.023 & 0.045 & 0.047 & 0.051 & 0.043 & 0.046 & 0.029 \\
\hline & & $10,10,10$ & 0.058 & 0.051 & 0.051 & 0.053 & 0.050 & 0.052 & 0.054 \\
\hline & & $18,27,45$ & 0.025 & 0.050 & 0.050 & 0.053 & 0.049 & 0.050 & 0.032 \\
\hline & & $30,30,30$ & 0.058 & 0.052 & 0.051 & 0.056 & 0.051 & 0.052 & 0.055 \\
\hline & & $60,90,150$ & 0.025 & 0.051 & 0.051 & 0.055 & 0.051 & 0.051 & 0.034 \\
\hline & & $100,100,100$ & 0.059 & 0.052 & 0.052 & 0.059 & 0.052 & 0.052 & 0.059 \\
\hline \multirow{12}{*}{$\begin{array}{l}\text { Skew Normal } \\
\qquad(\gamma=0.5)\end{array}$} & \multirow{6}{*}{$1,1,1$} & $6,9,15$ & 0.052 & 0.055 & 0.053 & 0.051 & 0.056 & 0.055 & 0.047 \\
\hline & & $10,10,10$ & 0.048 & 0.048 & 0.048 & 0.046 & 0.048 & 0.050 & 0.045 \\
\hline & & $18,27,45$ & 0.053 & 0.054 & 0.052 & 0.052 & 0.053 & 0.054 & 0.050 \\
\hline & & $30,30,30$ & 0.047 & 0.046 & 0.045 & 0.047 & 0.045 & 0.046 & 0.046 \\
\hline & & $60,90,150$ & 0.046 & 0.046 & 0.050 & 0.046 & 0.046 & 0.046 & 0.049 \\
\hline & & $100,100,100$ & 0.052 & 0.052 & 0.052 & 0.052 & 0.052 & 0.052 & 0.050 \\
\hline & \multirow{6}{*}{$1, \sqrt{2}, 2$} & $6,9,15$ & 0.026 & 0.052 & 0.051 & 0.053 & 0.052 & 0.053 & 0.032 \\
\hline & & $10,10,10$ & 0.059 & 0.051 & 0.051 & 0.054 & 0.051 & 0.053 & 0.052 \\
\hline & & $18,27,45$ & 0.023 & 0.051 & 0.053 & 0.055 & 0.050 & 0.051 & 0.038 \\
\hline & & $30,30,30$ & 0.053 & 0.046 & 0.046 & 0.052 & 0.046 & 0.046 & 0.057 \\
\hline & & $60,90,150$ & 0.022 & 0.048 & 0.052 & 0.052 & 0.048 & 0.048 & 0.052 \\
\hline & & $100,100,100$ & 0.058 & 0.050 & 0.053 & 0.058 & 0.050 & 0.050 & 0.082 \\
\hline \multirow{12}{*}{$\begin{array}{l}\text { Skew Normal } \\
\qquad(\gamma=-0.5)\end{array}$} & \multirow{6}{*}{$1,1,1$} & $6,9,15$ & 0.048 & 0.050 & 0.051 & 0.048 & 0.053 & 0.051 & 0.045 \\
\hline & & $10,10,10$ & 0.051 & 0.048 & 0.048 & 0.048 & 0.048 & 0.048 & 0.043 \\
\hline & & $18,27,45$ & 0.048 & 0.051 & 0.049 & 0.049 & 0.050 & 0.051 & 0.047 \\
\hline & & $30,30,30$ & 0.048 & 0.047 & 0.048 & 0.048 & 0.047 & 0.047 & 0.045 \\
\hline & & $60,90,150$ & 0.049 & 0.051 & 0.050 & 0.050 & 0.050 & 0.051 & 0.049 \\
\hline & & $100,100,100$ & 0.053 & 0.054 & 0.054 & 0.053 & 0.053 & 0.054 & 0.052 \\
\hline & \multirow{6}{*}{$1, \sqrt{2}, 2$} & $6,9,15$ & 0.024 & 0.047 & 0.049 & 0.048 & 0.047 & 0.049 & 0.031 \\
\hline & & $10,10,10$ & 0.058 & 0.051 & 0.051 & 0.054 & 0.050 & 0.052 & 0.053 \\
\hline & & $18,27,45$ & 0.024 & 0.049 & 0.049 & 0.053 & 0.048 & 0.049 & 0.037 \\
\hline & & $30,30,30$ & 0.054 & 0.048 & 0.048 & 0.052 & 0.047 & 0.048 & 0.059 \\
\hline & & $60,90,150$ & 0.022 & 0.050 & 0.050 & 0.054 & 0.049 & 0.050 & 0.054 \\
\hline & & $100,100,100$ & 0.058 & 0.054 & 0.055 & 0.058 & 0.054 & 0.054 & 0.085 \\
\hline
\end{tabular}


Table 3: Adjusted power results

\begin{tabular}{|c|c|c|c|c|c|c|c|c|c|c|}
\hline Distribution & $\sigma_{1}, \sigma_{2}, \sigma_{3}$ & $n_{1}, n_{2}, n_{3}$ & $\mu_{1}, \mu_{2}, \mu_{3}$ & $F$ & $F_{w}$ & $F_{w t}$ & $F_{B F}$ & $\chi_{A G}^{2}$ & $J$ & $\chi_{K W}^{2}$ \\
\hline \multirow{36}{*}{$\begin{array}{l}\text { Normal } \\
(\gamma=0)\end{array}$} & \multirow{18}{*}{$1,1,1$} & \multirow{3}{*}{$6,9,15$} & $0,0.25,0.5$ & 0.141 & 0.129 & 0.127 & 0.130 & 0.132 & 0.130 & 0.136 \\
\hline & & & $0,0.5,1$ & 0.447 & 0.400 & 0.392 & 0.417 & 0.404 & 0.399 & 0.428 \\
\hline & & & $0,1,2$ & 0.961 & 0.937 & 0.931 & 0.948 & 0.937 & 0.937 & 0.951 \\
\hline & & \multirow{3}{*}{$10,10,10$} & $0,0.25,0.5$ & 0.140 & 0.136 & 0.136 & 0.141 & 0.133 & 0.138 & 0.139 \\
\hline & & & $0,0.5,1$ & 0.449 & 0.436 & 0.436 & 0.451 & 0.430 & 0.437 & 0.433 \\
\hline & & & $0,1,2$ & 0.973 & 0.963 & 0.963 & 0.973 & 0.961 & 0.964 & 0.963 \\
\hline & & \multirow{3}{*}{$18,27,45$} & $0,0.25,0.5$ & 0.353 & 0.335 & 0.324 & 0.351 & 0.334 & 0.334 & 0.335 \\
\hline & & & $0,0.5,1$ & 0.911 & 0.897 & 0.886 & 0.905 & 0.895 & 0.896 & 0.894 \\
\hline & & & $0,1,2$ & 1.000 & 1.000 & 1.000 & 1.000 & 1.000 & 1.000 & 1.000 \\
\hline & & \multirow{3}{*}{$30,30,30$} & $0,0.25,0.5$ & 0.367 & 0.356 & 0.343 & 0.368 & 0.356 & 0.356 & 0.347 \\
\hline & & & $0,0.5,1$ & 0.937 & 0.930 & 0.915 & 0.937 & 0.929 & 0.930 & 0.920 \\
\hline & & & $0,1,2$ & 1.000 & 1.000 & 1.000 & 1.000 & 1.000 & 1.000 & 1.000 \\
\hline & & \multirow{3}{*}{$60,90,150$} & $0,0.25,0.5$ & 0.861 & 0.858 & 0.848 & 0.859 & 0.858 & 0.858 & 0.842 \\
\hline & & & $0,0.5,1$ & 1.000 & 1.000 & 1.000 & 1.000 & 1.000 & 1.000 & 1.000 \\
\hline & & & $0,1,2$ & 1.000 & 1.000 & 1.000 & 1.000 & 1.000 & 1.000 & 1.000 \\
\hline & & \multirow{3}{*}{$100,100,100$} & $0,0.25,0.5$ & 0.891 & 0.890 & 0.877 & 0.891 & 0.891 & 0.890 & 0.874 \\
\hline & & & $0,0.5,1$ & 1.000 & 1.000 & 1.000 & 1.000 & 1.000 & 1.000 & 1.000 \\
\hline & & & $0,1,2$ & 1.000 & 1.000 & 1.000 & 1.000 & 1.000 & 1.000 & 1.000 \\
\hline & \multirow{18}{*}{$1, \sqrt{2}, 2$} & \multirow{3}{*}{$6,9,15$} & $0,0.25,0.5$ & 0.091 & 0.093 & 0.089 & 0.086 & 0.096 & 0.093 & 0.087 \\
\hline & & & $0,0.5,1$ & 0.214 & 0.228 & 0.220 & 0.209 & 0.232 & 0.229 & 0.207 \\
\hline & & & $0,1,2$ & 0.682 & 0.721 & 0.703 & 0.691 & 0.724 & 0.723 & 0.677 \\
\hline & & \multirow{3}{*}{$10,10,10$} & $0,0.25,0.5$ & 0.084 & 0.089 & 0.089 & 0.086 & 0.087 & 0.089 & 0.082 \\
\hline & & & $0,0.5,1$ & 0.200 & 0.217 & 0.217 & 0.200 & 0.217 & 0.219 & 0.195 \\
\hline & & & $0,1,2$ & 0.657 & 0.697 & 0.697 & 0.655 & 0.702 & 0.700 & 0.647 \\
\hline & & \multirow{3}{*}{$18,27,45$} & $0,0.25,0.5$ & 0.164 & 0.192 & 0.186 & 0.175 & 0.192 & 0.193 & 0.170 \\
\hline & & & $0,0.5,1$ & 0.562 & 0.629 & 0.612 & 0.574 & 0.630 & 0.630 & 0.568 \\
\hline & & & $0,1,2$ & 0.995 & 0.997 & 0.996 & 0.996 & 0.997 & 0.997 & 0.995 \\
\hline & & \multirow{3}{*}{$30,30,30$} & $0,0.25,0.5$ & 0.167 & 0.185 & 0.180 & 0.166 & 0.187 & 0.185 & 0.165 \\
\hline & & & $0,0.5,1$ & 0.553 & 0.615 & 0.600 & 0.554 & 0.619 & 0.615 & 0.561 \\
\hline & & & $0,1,2$ & 0.996 & 0.997 & 0.997 & 0.996 & 0.998 & 0.997 & 0.994 \\
\hline & & \multirow{3}{*}{$60,90,150$} & $0,0.25,0.5$ & 0.491 & 0.559 & 0.545 & 0.496 & 0.558 & 0.560 & 0.496 \\
\hline & & & $0,0.5,1$ & 0.987 & 0.994 & 0.992 & 0.987 & 0.994 & 0.994 & 0.988 \\
\hline & & & $0,1,2$ & 1.000 & 1.000 & 1.000 & 1.000 & 1.000 & 1.000 & 1.000 \\
\hline & & \multirow{3}{*}{$100,100,100$} & $0,0.25,0.5$ & 0.483 & 0.553 & 0.545 & 0.482 & 0.554 & 0.553 & 0.483 \\
\hline & & & $0,0.5,1$ & 0.988 & 0.994 & 0.992 & 0.988 & 0.994 & 0.994 & 0.985 \\
\hline & & & $0,1,2$ & 1.000 & 1.000 & 1.000 & 1.000 & 1.000 & 1.000 & 1.000 \\
\hline \multirow{18}{*}{$\begin{array}{l}\text { Skew Normal } \\
\quad(\gamma=0.5)\end{array}$} & & & $0,0.25,0.5$ & 0.128 & 0.137 & 0.127 & 0.135 & 0.136 & 0.137 & 0.134 \\
\hline & & $6,9,15$ & $0,0.5,1$ & 0.419 & 0.395 & 0.372 & 0.410 & 0.392 & 0.393 & 0.418 \\
\hline & & & $0,1,2$ & 0.955 & 0.919 & 0.906 & 0.924 & 0.918 & 0.917 & 0.944 \\
\hline & & & $0,0.25,0.5$ & 0.150 & 0.141 & 0.141 & 0.149 & 0.138 & 0.141 & 0.144 \\
\hline & & $10,10,10$ & $0,0.5,1$ & 0.472 & 0.450 & 0.450 & 0.470 & 0.443 & 0.450 & 0.463 \\
\hline & & & $0,1,2$ & 0.972 & 0.964 & 0.964 & 0.971 & 0.961 & 0.964 & 0.967 \\
\hline & & & $0,0.25,0.5$ & 0.348 & 0.350 & 0.345 & 0.352 & 0.351 & 0.350 & 0.359 \\
\hline & $1,1,1$ & $18,27,45$ & $0,0.5,1$ & 0.907 & 0.891 & 0.887 & 0.895 & 0.892 & 0.891 & 0.907 \\
\hline & & & $0,1,2$ & 1.000 & 1.000 & 1.000 & 1.000 & 1.000 & 1.000 & 1.000 \\
\hline & & & $0,0.25,0.5$ & 0.397 & 0.395 & 0.386 & 0.397 & 0.396 & 0.395 & 0.398 \\
\hline & & $30,30,30$ & $0,0.5,1$ & 0.942 & 0.939 & 0.934 & 0.942 & 0.940 & 0.939 & 0.943 \\
\hline & & & $0,1,2$ & 1.000 & 1.000 & 1.000 & 1.000 & 1.000 & 1.000 & 1.000 \\
\hline & & & $0,0.25,0.5$ & 0.875 & 0.868 & 0.851 & 0.867 & 0.869 & 0.868 & 0.870 \\
\hline & & $60,90,150$ & $0,0.5,1$ & 1.000 & 1.000 & 1.000 & 1.000 & 1.000 & 1.000 & 1.000 \\
\hline & & & $0,1,2$ & 1.000 & 1.000 & 1.000 & 1.000 & 1.000 & 1.000 & 1.000 \\
\hline & & & $0,0.25,0.5$ & 0.890 & 0.887 & 0.882 & 0.890 & 0.887 & 0.887 & 0.894 \\
\hline & & $100,100,100$ & $0,0.5,1$ & 1.000 & 1.000 & 1.000 & 1.000 & 1.000 & 1.000 & 1.000 \\
\hline & & & $0,1,2$ & 1.000 & 1.000 & 1.000 & 1.000 & 1.000 & 1.000 & 1.000 \\
\hline
\end{tabular}


Table 4: Adjusted power results continued

\begin{tabular}{|c|c|c|c|c|c|c|c|c|c|c|}
\hline Distribution & $\sigma_{1}, \sigma_{2}, \sigma_{3}$ & $n_{1}, n_{2}, n_{3}$ & $\mu_{1}, \mu_{2}, \mu_{3}$ & $F$ & $F_{w}$ & $F_{w t}$ & $F_{B F}$ & $\chi_{A G}^{2}$ & $J$ & $\chi_{K W}^{2}$ \\
\hline \multirow{18}{*}{$\begin{array}{l}\text { Skew Normal } \\
\quad(\gamma=0.5)\end{array}$} & \multirow{18}{*}{$1, \sqrt{2}, 2$} & \multirow{3}{*}{$6,9,15$} & $0,0.25,0.5$ & 0.072 & 0.085 & 0.077 & 0.083 & 0.085 & 0.085 & 0.068 \\
\hline & & & $0,0.5,1$ & 0.181 & 0.216 & 0.193 & 0.199 & 0.215 & 0.216 & 0.172 \\
\hline & & & $0,1,2$ & 0.664 & 0.691 & 0.659 & 0.684 & 0.687 & 0.693 & 0.652 \\
\hline & & \multirow{3}{*}{$10,10,10$} & $0,0.25,0.5$ & 0.072 & 0.072 & 0.072 & 0.069 & 0.072 & 0.073 & 0.067 \\
\hline & & & $0,0.5,1$ & 0.180 & 0.194 & 0.194 & 0.176 & 0.194 & 0.192 & 0.170 \\
\hline & & & $0,1,2$ & 0.672 & 0.693 & 0.693 & 0.661 & 0.693 & 0.692 & 0.653 \\
\hline & & \multirow{3}{*}{$18,27,45$} & $0,0.25,0.5$ & 0.169 & 0.199 & 0.174 & 0.171 & 0.200 & 0.198 & 0.109 \\
\hline & & & $0,0.5,1$ & 0.573 & 0.625 & 0.591 & 0.579 & 0.627 & 0.625 & 0.475 \\
\hline & & & $0,1,2$ & 0.997 & 0.997 & 0.995 & 0.996 & 0.997 & 0.997 & 0.993 \\
\hline & & \multirow{3}{*}{$30,30,30$} & $0,0.25,0.5$ & 0.167 & 0.189 & 0.167 & 0.166 & 0.188 & 0.189 & 0.111 \\
\hline & & & $0,0.5,1$ & 0.584 & 0.639 & 0.593 & 0.582 & 0.638 & 0.639 & 0.483 \\
\hline & & & $0,1,2$ & 0.997 & 0.998 & 0.997 & 0.997 & 0.998 & 0.998 & 0.994 \\
\hline & & \multirow{3}{*}{$60,90,150$} & $0,0.25,0.5$ & 0.514 & 0.573 & 0.489 & 0.516 & 0.573 & 0.573 & 0.246 \\
\hline & & & $0,0.5,1$ & 0.991 & 0.994 & 0.989 & 0.991 & 0.994 & 0.994 & 0.949 \\
\hline & & & $0,1,2$ & 1.000 & 1.000 & 1.000 & 1.000 & 1.000 & 1.000 & 1.000 \\
\hline & & \multirow{3}{*}{$100,100,100$} & $0,0.25,0.5$ & 0.487 & 0.554 & 0.486 & 0.487 & 0.554 & 0.554 & 0.245 \\
\hline & & & $0,0.5,1$ & 0.990 & 0.994 & 0.990 & 0.990 & 0.994 & 0.994 & 0.946 \\
\hline & & & $0,1,2$ & 1.000 & 1.000 & 1.000 & 1.000 & 1.000 & 1.000 & 1.000 \\
\hline \multirow{36}{*}{$\begin{array}{l}\text { Skew Normal } \\
\qquad \gamma=-0.5)\end{array}$} & \multirow{18}{*}{$1,1,1$} & \multirow{3}{*}{$6,9,15$} & $0,0.25,0.5$ & 0.144 & 0.111 & 0.120 & 0.120 & 0.107 & 0.111 & 0.135 \\
\hline & & & $0,0.5,1$ & 0.432 & 0.364 & 0.386 & 0.396 & 0.355 & 0.365 & 0.417 \\
\hline & & & $0,1,2$ & 0.961 & 0.940 & 0.946 & 0.962 & 0.935 & 0.940 & 0.959 \\
\hline & & \multirow{3}{*}{$10,10,10$} & $0,0.25,0.5$ & 0.142 & 0.142 & 0.142 & 0.142 & 0.138 & 0.142 & 0.150 \\
\hline & & & $0,0.5,1$ & 0.455 & 0.449 & 0.449 & 0.457 & 0.439 & 0.451 & 0.470 \\
\hline & & & $0,1,2$ & 0.972 & 0.966 & 0.966 & 0.972 & 0.963 & 0.966 & 0.970 \\
\hline & & \multirow{3}{*}{$18,27,45$} & $0,0.25,0.5$ & 0.358 & 0.322 & 0.325 & 0.340 & 0.322 & 0.322 & 0.356 \\
\hline & & & $0,0.5,1$ & 0.917 & 0.908 & 0.903 & 0.920 & 0.907 & 0.908 & 0.921 \\
\hline & & & $0,1,2$ & 1.000 & 1.000 & 1.000 & 1.000 & 1.000 & 1.000 & 1.000 \\
\hline & & \multirow{3}{*}{$30,30,30$} & $0,0.25,0.5$ & 0.391 & 0.392 & 0.376 & 0.390 & 0.390 & 0.393 & 0.401 \\
\hline & & & $0,0.5,1$ & 0.941 & 0.939 & 0.929 & 0.941 & 0.937 & 0.939 & 0.943 \\
\hline & & & $0,1,2$ & 1.000 & 1.000 & 1.000 & 1.000 & 1.000 & 1.000 & 1.000 \\
\hline & & \multirow{3}{*}{$60,90,150$} & $0,0.25,0.5$ & 0.867 & 0.860 & 0.862 & 0.868 & 0.861 & 0.860 & 0.867 \\
\hline & & & $0,0.5,1$ & 1.000 & 1.000 & 1.000 & 1.000 & 1.000 & 1.000 & 1.000 \\
\hline & & & $0,1,2$ & 1.000 & 1.000 & 1.000 & 1.000 & 1.000 & 1.000 & 1.000 \\
\hline & & \multirow{3}{*}{$100,100,100$} & $0,0.25,0.5$ & 0.884 & 0.880 & 0.874 & 0.884 & 0.880 & 0.880 & 0.892 \\
\hline & & & $0,0.5,1$ & 1.000 & 1.000 & 1.000 & 1.000 & 1.000 & 1.000 & 1.000 \\
\hline & & & $0,1,2$ & 1.000 & 1.000 & 1.000 & 1.000 & 1.000 & 1.000 & 1.000 \\
\hline & & & $0,0.25,0.5$ & 0.101 & 0.094 & 0.098 & 0.092 & 0.091 & 0.094 & 0.102 \\
\hline & & $6,9,15$ & $0,0.5,1$ & 0.240 & 0.229 & 0.243 & 0.225 & 0.223 & 0.230 & 0.249 \\
\hline & & & $0,1,2$ & 0.672 & 0.720 & 0.728 & 0.691 & 0.707 & 0.720 & 0.694 \\
\hline & & & $0,0.25,0.5$ & 0.094 & 0.103 & 0.103 & 0.094 & 0.102 & 0.104 & 0.104 \\
\hline & & $10,10,10$ & $0,0.5,1$ & 0.220 & 0.240 & 0.240 & 0.220 & 0.242 & 0.242 & 0.243 \\
\hline & & & $0,1,2$ & 0.658 & 0.713 & 0.713 & 0.649 & 0.717 & 0.713 & 0.692 \\
\hline & & & $0,0.25,0.5$ & 0.188 & 0.197 & 0.207 & 0.178 & 0.197 & 0.197 & 0.242 \\
\hline & $1, \sqrt{2}, 2$ & $18,27,45$ & $0,0.5,1$ & 0.579 & 0.635 & 0.645 & 0.582 & 0.633 & 0.636 & 0.666 \\
\hline & & & $0,1,2$ & 0.993 & 0.999 & 0.999 & 0.996 & 0.999 & 0.999 & 0.998 \\
\hline & & & $0,0.25,0.5$ & 0.180 & 0.213 & 0.225 & 0.183 & 0.213 & 0.212 & 0.238 \\
\hline & & $30,30,30$ & $0,0.5,1$ & 0.574 & 0.646 & 0.658 & 0.576 & 0.647 & 0.646 & 0.659 \\
\hline & & & $0,1,2$ & 0.994 & 0.997 & 0.998 & 0.994 & 0.997 & 0.997 & 0.997 \\
\hline & & & $0,0.25,0.5$ & 0.518 & 0.576 & 0.624 & 0.517 & 0.577 & 0.576 & 0.659 \\
\hline & & $60,90,150$ & $0,0.5,1$ & 0.988 & 0.994 & 0.996 & 0.988 & 0.994 & 0.994 & 0.996 \\
\hline & & & $0,1,2$ & 1.000 & 1.000 & 1.000 & 1.000 & 1.000 & 1.000 & 1.000 \\
\hline & & & $0,0.25,0.5$ & 0.487 & 0.552 & 0.595 & 0.486 & 0.552 & 0.552 & 0.630 \\
\hline & & $100,100,100$ & $0,0.5,1$ & 0.984 & 0.992 & 0.994 & 0.984 & 0.992 & 0.991 & 0.995 \\
\hline & & & $0,1,2$ & 1.000 & 1.000 & 1.000 & 1.000 & 1.000 & 1.000 & 1.000 \\
\hline
\end{tabular}

